\title{
Metales pesados en suelos y sedimentos de la zona cañera del sur de Quintana Roo, México
}

\section{Gibrán Eduardo Tun-Canto ${ }^{1, *}$, Teresa Álvarez-Legorreta ${ }^{1}$, Gonzalo Zapata-Buenfil ${ }^{2}$ y Eloy Sosa-Cordero ${ }^{1}$}

\author{
${ }^{1}$ El Colegio de la Frontera Sur, Av. Centenario $\mathrm{km}$ 5.5, sin número exterior, sin cruzamientos, \\ Col. Pacto Obrero Campesino, C.P. 77014, Chetumal, Quintana Roo, México. \\ ${ }^{2}$ Instituto Nacional de Investigaciones, Forestales Agrícolas y Pecuarias, \\ km 25 carretera Chetumal-Bacalar, C.P. 77900, Chetumal, Quintana Roo, México. \\ *getc2302@gmail.com
}

\section{RESUMEN}

Con el objetivo de determinar la presencia y distribución de los metales pesados en la zona cañera de la cuenca del Río Hondo, sur de Quintana Roo, México, se colectaron 10 muestras de sedimentos superficiales del canal de desagüe principal y 18 muestras de suelos superficiales desarrollados sobre un acuífero kárstico en las parcelas aledañas al canal. Las muestras de ambas matrices ambientales fueron tomadas en febrero de 2016, durante la temporada de secas. Se determinaron las concentraciones totales de $\mathrm{Hg}, \mathrm{Cd}, \mathrm{Cu}$ y Fe, y valores fisicoquímicos como el $\mathrm{pH}$, materia orgánica y la capacidad de intercambio catiónico de los suelos. Para comprender la distribución de los metales pesados se obtuvieron parámetros estadísticos descriptivos; además, se hicieron correlaciones de Pearson, análisis de varianza, pruebas $t$ de Student, análisis de componentes principales y de dendrogramas. Las concentraciones promedio de metales pesados encontradas en suelo y sedimentos en orden descendente fueron: $\mathrm{Fe}>\mathrm{Cu}>\mathrm{Cd}>\mathrm{Hg}$ con valores en suelo de $25,334.13 \mathrm{mg} \cdot \mathrm{kg}^{-1}, 10.56 \mathrm{mg} \cdot \mathrm{kg}^{-1}, 0.55 \mathrm{mg} \cdot \mathrm{kg}^{-1} \mathrm{y}$ $0.02 \mathrm{mg} \cdot \mathrm{kg}^{-1}$, respectivamente; y para sedimento los valores obtenidos fueron $19,952.68 \mathrm{mg} \cdot \mathrm{kg}^{-1}, 8.00 \mathrm{mg} \cdot \mathrm{kg}^{-1}, 0.54 \mathrm{mg} \cdot \mathrm{kg}^{-1} \mathrm{y} 0.06 \mathrm{mg} \cdot \mathrm{kg}^{-1}$, respectivamente. Los valores de los parámetros fisicoquímicos $(\mathrm{pH}$, materia orgánica y capacidad de intercambio catiónico) resultaron homogéneos dentro del área de estudio. Las concentraciones detectadas de $\mathrm{Hg}, \mathrm{Cd}$ y $\mathrm{Cu}$ podrían provenir de fuentes antropogénicas. Se determinó que el Fe se encuentra de manera natural en el área analizada y su distribución posiblemente ha sido afectada por la actividad agrícola. El factor que determina la distribución de los metales pesados en los suelos agrícolas de la zona cañera es el arrastre de partículas de suelo causado por los escurrimientos, y el mayor aporte de estos contaminantes al drenaje agrícola ocurre principalmente por el arrastre de partículas provenientes de suelos de tipo gleysol.

Palabras clave: Metales pesados; karst; contaminación del suelo; zona cañera; Río Hondo; México.

\begin{abstract}
In order to determine the heavy metals content and distribution in an area cultivated with sugarcane in the Río Hondo basin, southern Quintana Roo, Mexico, 10 samples of surficial sediments were collected from the main agricultural drain and 18 samples from soils developed on a karst aquifer. The samples were obtained on february 2016, during the dry season. Concentrations of $\mathrm{Hg}, \mathrm{Cd}, \mathrm{Cu}$, and $\mathrm{Fe}$ and physicochemical parameters such $p H$, organic matter and cation exchange capacity were measured. In order to understand the distribution of the heavy metals, we performed descriptive statistics, Pearson correlation, analysis of variance, $t$ test, principal component analysis and hierarchical clustering analysis. The mean concentration of heavy metals in soil, in descending order were: $\mathrm{Fe}>\mathrm{Cu}>\mathrm{Cd}>\mathrm{Hg}$ with soil values of 25,334.13 $\mathrm{mg} \cdot \mathrm{kg}^{-1}, 10.56 \mathrm{mg} \cdot \mathrm{kg}^{-1}$, $0.55 \mathrm{mg} \cdot \mathrm{kg}^{-1}$ and $0.02 \mathrm{mg} \cdot \mathrm{kg}^{-1}$, respectively; the sediment values found were $19,952.68 \mathrm{mg} \cdot \mathrm{kg}^{-1}, 8.00 \mathrm{mg} \cdot \mathrm{kg}^{-1}, 0.54 \mathrm{mg} \cdot \mathrm{kg}^{-1}$ and $0.06 \mathrm{mg} \cdot \mathrm{kg}^{-1}$, respectively. The physical and chemical parameters were homogeneous within the study area. The origin of $\mathrm{Hg}, \mathrm{Cd}$ and $\mathrm{Cu}$ concentrations could be anthropogenic. Although Fe is naturally found in the study area, it is possible that the current distribution of this metal is also due to agricultural activity. The main factor in the distribution of heavy metals in the agricultural soils of the sugarcane zone is the carrying of soil particulates caused by runoff, and the greater contribution of these pollutants to the agricultural drain occurs mainly by the entrainment of particulates from gleysol type soils.
\end{abstract}

Key words: Heavy metals; karst; soil contamination; sugar cane zone; Río Hondo; Mexico.

\section{INTRODUCCIÓN}

México se encuentra dividido en 37 regiones hidrológicas que a su vez se agrupan en 13 regiones hidrológico-administrativas. Las aguas del estado de Quintana Roo se ubican en la región hidrológica-

Tun-Canto, G.E., Teresa Álvarez-Legorreta, T., Zapata-Buenfil, G., Sosa-Cordero, E., 2017, Metales pesados en suelos y sedimentos de la zona cañera del sur de Quintana Roo, México: Revista Mexicana de Ciencias Geológicas, v. 34, núm. 3, p. 157-169. 
administrativa llamada "Península de Yucatán" y dentro de la región hidrológica 33 llamada "Yucatán Este” (CONAGUA, 2010). El Río Hondo, la frontera natural entre México y Belice, se encuentra dentro de la subcuenca transfronteriza Río Hondo (RH33-Ad), que forma parte de la cuenca bahía de Chetumal y otras (RH33-Ac) (INEGI, 2013a). La cuenca del Río Hondo cuenta con una superficie de 13465 $\mathrm{km}^{2}$, de los cuales el $57 \%$ corresponde a México, $22 \%$ a Belice y el $21 \%$ restante a Guatemala (CCPY y CONAGUA, 2012). En las riberas de México y Belice se desarrollan actividades agrícolas, con el cultivo de grandes extensiones de caña de azúcar (Saccharum officinarum). En la ribera mexicana se encuentra el ingenio azucarero San Rafael de Pucté fundado en 1972. El ingenio recibe la producción de caña de azúcar cultivada por ejidatarios y pequeños propietarios en una superficie de 34,848 ha (SIAP, 2014). El área de cultivo requiere el uso de grandes cantidades de agroquímicos, lo cual constituye una fuente potencial de contaminación para los suelos, aguas superficiales y el manto freático de la cuenca, debido a su transporte a través de canales agrícolas secundarios. Los canales fueron construidos con el fin de evitar la inundación de las parcelas de cultivo, los cuales se conectan con un canal principal que descarga sus aguas en el Río Hondo. El río, que a su vez desemboca al sur de la bahía de Chetumal, podría arrastrar los residuos de la zona cañera poniendo en riesgo la fauna acuática y la salud humana.

En la agricultura, el suelo se modifica física y químicamente para satisfacer la demanda de nutrientes que los diversos cultivos necesitan para desarrollarse. También, el suelo es el principal receptor de los residuos agroquímicos (plaguicidas, fertilizantes, metales pesados, etc.), así como el conductor de éstos al manto acuífero. Los residuos de los agroquímicos representan un riesgo, en particular, para las aguas subterráneas debido a que los suelos de la zona se desarrollan sobre un acuífero kárstico maduro con amplias fisuras, fracturas y cuevas (Sánchez-Sánchez et al., 2015). Las características del acuífero se reflejan en una alta conductividad hidráulica, haciéndolos altamente vulnerables a la contaminación (Herrera, 2011). Un acuífero kárstico se divide en cuatro zonas: zona de absorción o recarga (epikarst), zona de circulación vadosa (no saturada o de saturación vertical), zona de fluctuación o epifreática y zona freática o saturada (Aguilar-Duarte et al., 2013). En este estudio la zona de interés es la de absorción o recarga la cual incluye al suelo $y$ al relieve.

Los metales pesados (MP) son todos aquellos elementos metálicos y metaloides que causan un impacto ambiental debido a su toxicidad (Doménech y Peral, 2006). Como elementos traza, algunos MP como el cobre $(\mathrm{Cu})$, selenio $(\mathrm{Se})$, zinc $(\mathrm{Zn})$ son esenciales para mantener un correcto metabolismo en los seres vivos y, en particular, para el cuerpo humano. Sin embargo, en concentraciones relativamente altas pueden causar intoxicación y los síntomas específicos dependerán del elemento. Los MP no esenciales como cadmio $(\mathrm{Cd})$, plomo $(\mathrm{Pb})$, mercurio $(\mathrm{Hg})$ y metaloides como el arsénico (As) son altamente tóxicos para los humanos, plantas o vida silvestre (Prieto-Méndez et al., 2009). En el suelo los MP están distribuidos en las fases sólidas (arcillas y minerales) y líquidas (agua intersticial) y se pueden encontrar en diferentes fracciones o especies, tales como la fracción intercambiable, unida a carbonatos, unida a óxidos de Fe y Mn o reducible, unida a materia orgánica u oxidable y residual (Tessier et al., 1979; McLean y Bledsoe, 1992). Su especiación en cada fase depende de diferentes factores como la unión con complejos orgánicos e inorgánicos, reacciones de reducción-oxidación, hidrólisis, adsorción, intercambio de iones y fenómenos de disolución, precipitación o quelación (Kennou et al., 2015). Cada interacción química les confiere diferente comportamiento físico y químico, modificando así su movilidad, disponibilidad biológica y toxicidad. Al mismo tiempo, estos factores pueden ser afectados por las propiedades fisicoquímicas del suelo como la variación en el $\mathrm{pH}$ (siendo éste el más importante en la disposición de los MP), el contenido de materia orgánica (MO), la capacidad de intercambio catiónico (CIC) y la textura (FAR y PPIC, 1988; De Matos et al., 2001; Dube et al., 2001; Violante et al., 2010; Sherene, 2010; Kennou et al., 2015). Cuando los MP están disueltos en agua, la conductividad hidráulica resultante de la humedad y la porosidad del suelo, pueden influir directamente en la movilidad.

El monitoreo de los niveles de MP en suelos agrícolas, en especial los desarrollados sobre acuíferos kársticos, es importante para evaluar y conservar la calidad de los mismos, así como de las aguas superficiales y subterráneas, y de esta manera prevenir problemas de salud en humanos y en la vida silvestre. La falta de información sobre la presencia, distribución y movilidad de los MP vertidos en la zona cañera del sur de Quintana Roo, dificulta la toma de decisiones para atender los problemas de contaminación por metales pesados detectados en la subcuenca Río Hondo. Es por ello que este estudio tiene como objetivos evaluar la concentración de $\mathrm{Hg}, \mathrm{Cd}, \mathrm{Cu}$ y $\mathrm{Fe}$ en suelos y sedimentos del drenaje principal de la zona cañera, así como determinar su distribución y relación con los parámetros fisicoquímicos del suelo.

\section{Antecedentes}

Fernández-Pérez y Leal-Bautista (2013), evaluaron la concentración de plaguicidas en suelos de la zona cañera a cuatro profundidades, así como en lixiviados y agua subterránea (pozos). En el suelo registraron la presencia de plaguicidas organoclorados, organofosforados y piretroides con mayor incidencia a una profundidad de 0 a $45 \mathrm{~cm}$. En los lixiviados detectaron organofosforados y piretroides, indicando que estos compuestos eran arrastrados por el agua hacia el manto acuífero. Los resultados obtenidos en agua subterránea corroboraron la lixiviación de los agroquímicos, ya que se detectaron los diferentes grupos de compuestos. Sánchez-Pool (2007) en un estudio sobre MP ( $\mathrm{Cd}, \mathrm{Cu}, \mathrm{Fe}, \mathrm{Pb}$ y $\mathrm{Zn}$ ) en agua, sedimento y macro-algas para la bahía de Chetumal, reportó que las concentraciones de $\mathrm{Fe} \mathrm{y} \mathrm{Pb}$, especialmente en agua, fueron mayores en la temporada de lluvias y concluyó que las fuentes principales son las zonas urbanas y/o recreativas, así como el aporte proveniente de las zonas de cultivo de las riberas del Río Hondo. Para este sistema también se realizó un estudio de MP en tejido óseo de la especie Trichechus manatus donde se reportaron concentraciones de $\mathrm{Cd}, \mathrm{Cr}, \mathrm{Cu}, \mathrm{Pb}, \mathrm{Ni}, \mathrm{Zn}$ y As (Romero-Calderón et al., 2016). Recientemente Buenfil-Rojas et al. (2015) detectaron niveles de Cd, Hg y metalotioneínas en tejidos (crestas) y plasma sanguíneo del cocodrilo de pantano (Crocodylus moreletii) que habita en el Río Hondo, sugiriendo que una de las principales fuentes de exposición a estos contaminantes podrían ser los productos químicos utilizados en las actividades agrícolas que se realizan en las riberas de México y Belice.

\section{METODOLOGÍA}

\section{Área de estudio}

El área de estudio se ubica en la zona de abastecimiento del ingenio San Rafael de Pucté, localizado en el sur de Quintana Roo (Figura 1), a $6 \mathrm{~km}$ al oeste del poblado Álvaro Obregón $\left(18^{\circ} 14^{\prime} 37.77^{\prime \prime} \mathrm{N}\right)$ y $\left.88^{\circ} 40^{\prime} 03.89^{\prime \prime} \mathrm{W}\right)$ y a $9 \mathrm{~km}$ del poblado Pucté $\left(18^{\circ} 14^{\prime} 07.77^{\prime \prime} \mathrm{N}\right.$ y $\left.88^{\circ} 40^{\prime} 06.75^{\prime \prime} \mathrm{W}\right)$.

\section{Muestreo}

El muestreo se realizó en febrero de 2016, que corresponde a la temporada de secas. El área de estudio se dividió en tres zonas (A, B y C) tomando como criterio la ubicación geográfica del canal principal de desagüe de la zona cañera y la altitud (Figura 1) (Tablas 1 y 2). La 


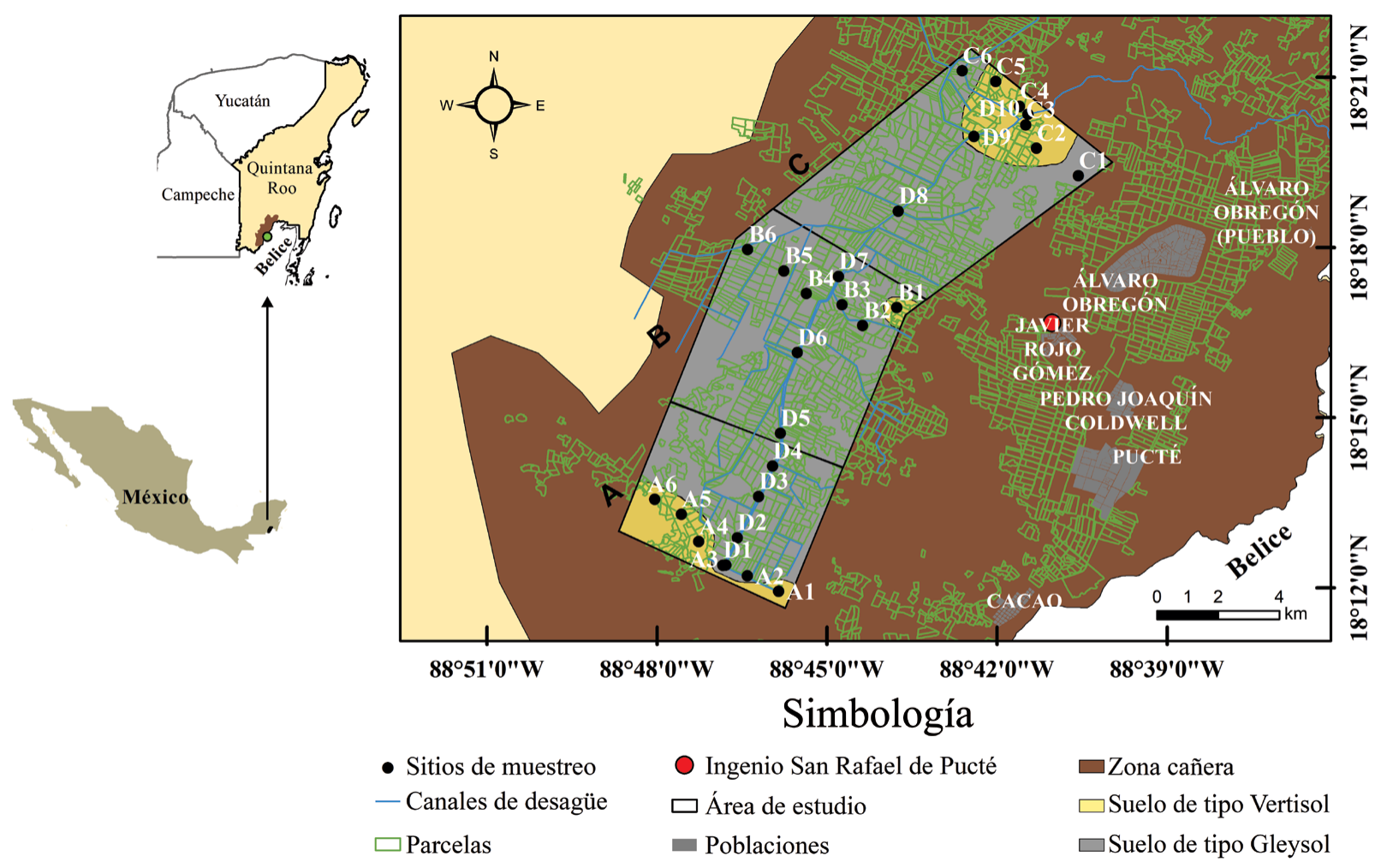

Figura 1. Mapa de ubicación geográfica del área de estudio. Las coordenadas geográficas se muestran en grados. El Datum es WGS 1984.

zona A se localiza en el sur y es la parte más alta (inicio del canal), con promedio de altitud de $55 \mathrm{~m}$; la zona B corresponde a la parte media y de altitud homogénea, con $30 \mathrm{~m}$ en promedio y la zona $\mathrm{C}$ en el norte, con altitudes intermedias (final del canal), en promedio de $35.92 \mathrm{~m}$. Se seleccionaron 18 sitios de muestreo para suelos agrícolas y 10 sitios en el canal principal de desagüe para sedimentos. Para obtener muestras de suelo los sitios se distribuyeron en ambos lados del canal. Tres sitios con dirección noroeste y tres al sureste, con una distancia de $1.5 \mathrm{~km}$ aproximadamente entre cada punto, ubicados en suelos de tipo gleysol $(n=9)$ y vertisol $(n=9)$ (Tabla 1) que son los más representativos de la zona bajo estudio (Figura 1). Las muestras de sedimento fueron tomadas en sitios cercanos a los puentes distribuidos a lo largo del canal (Figura 1).

Cada muestra de suelo se colectó por triplicado con una barrena

Tabla 1. Concentración de metales pesados y parámetros fisicoquímicos en suelos.

\begin{tabular}{|c|c|c|c|c|c|c|c|c|c|c|c|}
\hline \multirow[t]{2}{*}{ Sitio } & \multicolumn{2}{|c|}{ Coordenadas } & \multirow{2}{*}{$\begin{array}{l}\text { Altitud } \\
(\mathrm{m})\end{array}$} & \multirow[t]{2}{*}{ Suelo } & \multirow{2}{*}{$\underset{\left(\mu \mathrm{g} \cdot \mathrm{kg}^{1}\right)}{\mathbf{H g}}$} & \multirow{2}{*}{$\begin{array}{c}\text { Cd } \\
\left(\mathrm{mg} \cdot \mathrm{kg}^{1}\right)\end{array}$} & \multirow{2}{*}{$\underset{\left(\mathrm{mg} \cdot \mathrm{kg}^{1}\right)}{\mathrm{Cu}}$} & \multirow{2}{*}{$\begin{array}{c}\mathrm{Fe} \\
\left(\mathrm{mg} \cdot \mathrm{kg}^{1}\right)\end{array}$} & \multirow[t]{2}{*}{$\mathrm{pH}$} & \multirow{2}{*}{$\begin{array}{l}\text { MO } \\
(\%)\end{array}$} & \multirow{2}{*}{$\begin{array}{c}\text { CIC } \\
\left(\mathrm{cmol} \cdot \mathrm{kg}^{-1}\right)\end{array}$} \\
\hline & Latitud N & Longitud W & & & & & & & & & \\
\hline A1 & $18^{\circ} 11^{\prime} 56.5^{\prime \prime}$ & $88^{\circ} 45^{\prime} 51.1^{\prime \prime}$ & 60.00 & Vertisol & ND & 0.24 & 11.39 & $19,391.73$ & 7.55 & 0.73 & 44.7 \\
\hline A2 & $18^{\circ} 12^{\prime} 12.9^{\prime \prime}$ & $88^{\circ} 46^{\prime} 24.2^{\prime \prime}$ & 49.07 & Gleysol & ND & 0.2 & 12.16 & $15,397.43$ & 5.87 & 0.52 & 44.5 \\
\hline A3 & $18^{\circ} 12^{\prime} 24.1^{\prime \prime}$ & $88^{\circ} 46^{\prime} 47.0^{\prime \prime}$ & 45.00 & Gleysol & ND & 0.16 & 6.03 & $16,996.61$ & 6.27 & 0.83 & 44.9 \\
\hline A4 & $18^{\circ} 12^{\prime} 48.9^{\prime \prime}$ & $88^{\circ} 47^{\prime} 15.7^{\prime \prime}$ & 54.95 & Vertisol & 10.01 & ND & 6.2 & $13,573.23$ & 7.04 & 0.67 & 44.8 \\
\hline A5 & $18^{\circ} 13^{\prime} 17.9^{\prime \prime}$ & $88^{\circ} 47^{\prime} 34.0^{\prime \prime}$ & 57.80 & Vertisol & ND & 0.05 & 5.76 & $10,346.97$ & 8.09 & 0.78 & 44.4 \\
\hline A6 & $18^{\circ} 13^{\prime} 33.6^{\prime \prime}$ & $88^{\circ} 48^{\prime} 02.2^{\prime \prime}$ & 75.00 & Vertisol & ND & 0.12 & 7.7 & $16,363.72$ & 7.91 & 0.94 & 44.6 \\
\hline B1 & $18^{\circ} 16^{\prime} 56.5^{\prime \prime}$ & $88^{\circ} 43^{\prime} 46.0^{\prime \prime}$ & 30.00 & Vertisol & 160 & 0.64 & 12.06 & $53,624.1$ & 6.57 & 0.81 & 45.0 \\
\hline B2 & $18^{\circ} 16^{\prime} 37.4^{\prime \prime}$ & $88^{\circ} 44^{\prime} 22.3^{\prime \prime}$ & 30.00 & Gleysol & 3.80 & 1 & 14.66 & $51,712.4$ & 5.87 & 0.74 & 44.8 \\
\hline B3 & $18^{\circ} 16^{\prime} 59.4^{\prime \prime}$ & $88^{\circ} 44^{\prime} 43.9^{\prime \prime}$ & 30.00 & Gleysol & 33.26 & 0.97 & 14.88 & $42,028.74$ & 5.91 & 0.89 & 43.2 \\
\hline B4 & $18^{\circ} 17^{\prime} 11.3^{\prime \prime}$ & $88^{\circ} 45^{\prime} 21.9^{\prime \prime}$ & 30.00 & Gleysol & 53.13 & 0.69 & 4.2 & $14,690.29$ & 5.56 & 0.78 & 44.9 \\
\hline B5 & $18^{\circ} 17^{\prime} 35.2^{\prime \prime}$ & $88^{\circ} 45^{\prime} 45.6^{\prime \prime}$ & 30.00 & Gleysol & 13.00 & 0.81 & 0.9 & $8,786.5$ & 5.23 & 0.69 & 44.7 \\
\hline B6 & $18^{\circ} 17^{\prime} 57.8^{\prime \prime}$ & $88^{\circ} 46^{\prime} 24.0^{\prime \prime}$ & 30.00 & Gleysol & 18.81 & 0.93 & 5.8 & $13,363.95$ & 5.64 & 0.77 & 44.4 \\
\hline $\mathrm{C} 1$ & $18^{\circ} 19^{\prime} 15.8^{\prime \prime}$ & $88^{\circ} 40^{\prime} 33.9^{\prime \prime}$ & 40.74 & Gleysol & 9.62 & 0.56 & 23.84 & $45,029.31$ & 6.5 & 0.96 & 43.6 \\
\hline $\mathrm{C} 2$ & $18^{\circ} 19^{\prime} 44.8^{\prime \prime}$ & $88^{\circ} 41^{\prime} 18.2^{\prime \prime}$ & 44.06 & Vertisol & 40.34 & 0.91 & 15.17 & $9,022.92$ & 6.41 & 0.72 & 44.5 \\
\hline $\mathrm{C} 3$ & $18^{\circ} 20^{\prime} 09.5^{\prime \prime}$ & $88^{\circ} 41^{\prime} 29.7$ '” & 30.00 & Vertisol & 29.09 & 0.57 & 17.56 & $30,045.42$ & 7.72 & 1.05 & 44.5 \\
\hline $\mathrm{C} 4$ & $18^{\circ} 20^{\prime} 29.2^{\prime \prime}$ & $88^{\circ} 41^{\prime} 36.2^{\prime \prime}$ & 37.35 & Vertisol & 35.17 & 0.65 & 10.31 & $19,573.84$ & 7.61 & 0.82 & 44.7 \\
\hline $\mathrm{C} 5$ & $18^{\circ} 20^{\prime} 55.4^{\prime \prime}$ & $88^{\circ} 42^{\prime} 01.4^{\prime \prime}$ & 39.33 & Vertisol & 16.32 & 0.69 & 9.57 & $35,101.57$ & 5.83 & 0.83 & 44.6 \\
\hline C6 & $18^{\circ} 21^{\prime} 06.9^{\prime \prime}$ & $88^{\circ} 42^{\prime} 36.7^{\prime \prime}$ & 30.00 & Gleysol & 9.48 & 0.67 & 12.06 & $40,965.77$ & 6.07 & 0.89 & 43.6 \\
\hline
\end{tabular}

$\mathrm{ND}=$ No detectado. 
Tabla 2. Concentración de metales pesados y parámetros fisicoquímicos en sedimentos.

\begin{tabular}{|c|c|c|c|c|c|c|c|c|c|c|}
\hline \multirow[t]{2}{*}{ Sitio } & \multicolumn{2}{|c|}{ Coordenadas } & \multirow{2}{*}{$\begin{array}{c}\text { Altitud } \\
(\mathrm{km})\end{array}$} & \multirow{2}{*}{$\begin{array}{c}\text { Distancia } \\
(\mathrm{m})\end{array}$} & \multirow{2}{*}{$\underset{\left(\mu \mathrm{g} \cdot \mathrm{kg}^{1}\right)}{\mathbf{H g}}$} & \multirow{2}{*}{$\begin{array}{c}\text { Cd } \\
\left(\mathrm{mg} \cdot \mathrm{kg}^{1}\right)\end{array}$} & \multirow{2}{*}{$\begin{array}{c}\mathrm{Cu} \\
\left(\mathrm{mg} \cdot \mathrm{kg}^{1}\right)\end{array}$} & \multirow{2}{*}{$\begin{array}{c}\mathbf{F e} \\
\left(\mathrm{mg} \cdot \mathrm{kg}^{1}\right)\end{array}$} & \multirow[t]{2}{*}{$\mathrm{pH}$} & \multirow{2}{*}{$\begin{array}{l}\text { MO } \\
(\%)\end{array}$} \\
\hline & Latitud N & Longitud W & & & & & & & & \\
\hline D1 & $18^{\circ} 12^{\prime} 23.9^{\prime \prime}$ & $88^{\circ} 46^{\prime} 50.2^{\prime \prime}$ & 45.00 & 0 & 127.26 & 0.52 & 19.82 & $23,131.99$ & 8.11 & 0.59 \\
\hline $\mathrm{D} 2$ & $18^{\circ} 12^{\prime} 53.0^{\prime \prime}$ & $88^{\circ} 46^{\prime} 34.9^{\prime \prime}$ & 45.00 & 1.08 & 99.75 & 0.56 & 5.79 & $16,115.52$ & 8.08 & 0.45 \\
\hline D3 & $18^{\circ} 13^{\prime} 36.7^{\prime \prime}$ & $88^{\circ} 46^{\prime} 12.3^{\prime \prime}$ & 45.00 & 2.56 & 78.85 & 0.6 & 4.91 & $16,573.30$ & 8.08 & 0.49 \\
\hline $\mathrm{D} 4$ & $18^{\circ} 14^{\prime} 08.8^{\prime \prime}$ & $88^{\circ} 45^{\prime} 57.4^{\prime \prime}$ & 37.51 & 3.62 & 39.50 & 0.64 & 2.77 & $11,064.19$ & 7.57 & 0.48 \\
\hline D5 & $18^{\circ} 14^{\prime} 43.5^{\prime \prime}$ & $88^{\circ} 45^{\prime} 49.4^{\prime \prime}$ & 30.00 & 4.80 & 87.19 & 0.73 & 11.43 & $46,261.53$ & 8.00 & 0.49 \\
\hline D6 & $18^{\circ} 16^{\prime} 08.8^{\prime \prime}$ & $88^{\circ} 45^{\prime} 31.4^{\prime \prime}$ & 30.00 & 7.50 & 60.05 & 0.75 & 13.78 & $27,181.49$ & 7.61 & 0.62 \\
\hline D7 & $18^{\circ} 17^{\prime} 29.3^{\prime \prime}$ & $88^{\circ} 44^{\prime} 47.7^{\prime \prime}$ & 30.00 & 10.36 & 62.75 & 0.59 & 8.12 & $14,498.53$ & 7.76 & 0.66 \\
\hline D8 & $18^{\circ} 18^{\prime} 38.4^{\prime \prime}$ & $88^{\circ} 43^{\prime} 44.4^{\prime \prime}$ & 30.00 & 13.24 & 50.71 & 0.31 & 6.48 & $13,625.95$ & 7.85 & 0.57 \\
\hline D9 & $18^{\circ} 19^{\prime} 57.3^{\prime \prime}$ & $88^{\circ} 42^{\prime} 24.4^{\prime \prime}$ & 25.83 & 16.71 & 27.72 & 0.37 & 2.05 & $14,803.00$ & 8.05 & 0.51 \\
\hline D10 & $18^{\circ} 20^{\prime} 20.9^{\prime \prime}$ & $88^{\circ} 41^{\prime} 27.2^{\prime \prime}$ & 30.00 & 18.68 & 5.96 & 0.3 & 4.81 & $16,271.3$ & 7.89 & 0.56 \\
\hline
\end{tabular}

de acero inoxidable en suelos superficiales ( $0-0.2 \mathrm{~m}$ de profundidad) dentro de un área de aproximadamente $100 \mathrm{~m}^{2}$ en parcelas cultivadas con caña de azúcar. Los sedimentos superficiales se colectaron por triplicado (una muestra en cada orilla y una en el centro del canal) con una pala de plástico rígido. Las muestras de cada sustrato se colocaron en bolsas de plástico para su transporte y almacenamiento en refrigerador a una temperatura de $4{ }^{\circ} \mathrm{C}$. Los triplicados de muestras fueron mezclados para sus respectivos análisis.

\section{Acondicionamiento de muestras}

Todo el material usado en los análisis fue lavado con ácido nítrico $\left(\mathrm{HNO}_{3}\right)$ grado absorción atómica al $10 \%$. El agua usada para diluciones y preparación de reactivos fue de tipo I (desionizada).

Las muestras de suelo fueron secadas a $60^{\circ} \mathrm{C}$ en una estufa durante $72 \mathrm{~h}$. Las muestras de sedimento se secaron en una liofilizadora (marca $\left.\mathrm{LABCONCO}^{\oplus}\right)$. Una vez secas, se molieron y pasaron por un tamiz número 35 (marca Fisher Scientific Company) para obtener un tamaño de partícula de $500 \mu \mathrm{m}$.

\section{Determinación de parámetros fisicoquímicos}

El pH se determinó con el método AS-02 "Determinación del pH del suelo medido en solución acuosa" con potenciómetro (marca OAKTON, modelo pH 510 series) y la capacidad de intercambio catiónico (CIC) en suelos con el método AS-13 "Determinación de CIC en suelos ácidos y calcáreos con tiourea de plata", ambos incluidos en la NOM-021-SEMARNAT-2000 (SEMARNAT, 2002). El contenido de materia orgánica $(\mathrm{MO})$ se determinó por pérdida de peso al incinerar con una adaptación del método de Storer (1984), que consistió en eliminar contenido de carbonatos al agregar solución de ácido clorhídrico $1 \mathrm{~N}$ hasta que no se observó efervescencia .

\section{Determinación de metales pesados}

Como parte del control de calidad se procesaron triplicados de material de referencia certificado (MRC) de sedimentos de agua dulce (Sediment 3 marca FLUKA Analytical), de los cuales se obtuvieron los siguientes porcentajes de recuperación: $\mathrm{Hg}=104.13 \%, \mathrm{Cd}=89.28 \%$, $\mathrm{Cu}=70.12 \%$ y $\mathrm{Fe}=99.176 \%$. También se procesaron blancos de procedimiento, los cuales tuvieron valores cercanos a cero.

Los MP totales fueron extraídos en horno de microondas (marca CEM, modelo Mars 6) (Mangum, 2009). La determinación de los elementos se realizó por espectrofotometría de absorción atómica con un espectrofotómetro marca GBC modelo AVANTA PM. La lectura de $\mathrm{Cd}, \mathrm{Cu}$ y Fe se realizó por aspiración directa con quemador de flama aire-acetileno con límites de detección para $\mathrm{Cd}$ y $\mathrm{Cu}$ de 0.045 y $0.201 \mathrm{mg} \cdot \mathrm{l}^{-1}$, respectivamente. El Hg se leyó con accesorio generador de hidruros (modelo GBC HG-3000) con límite de detección de $0.462 \mu \mathrm{g} \cdot \mathrm{l}^{-1}$. Previo a las lecturas de Hg la curva de calibración, muestras, blancos y MRC se sometieron a un tratamiento de oxidación con permanganato de potasio $\left(\mathrm{KMnO}_{4}\right)$ al $5 \%$.

\section{Análisis estadísticos}

Todos los análisis estadísticos que a continuación se describen se realizaron con el programa R versión 3.1.3 (Murdock, 2014). En el caso de los sitios donde no se detectaron los elementos, el valor fue sustituido por el equivalente a la mitad del límite de detección del método para el elemento correspondiente (Rainwater et al., 2007).

Inicialmente fueron calculados los parámetros estadísticos básicos (promedio, mínimo, máximo, desviación estándar (DE) y coeficientes de variación $(\mathrm{CV})$ de las variables de respuesta y covariables consideradas. Se calculó la correlación de Pearson para cada matriz, y se realizó el análisis de componentes principales (ACP) y de dendrogramas (AC) con el fin de encontrar los factores que se pudieran asociar con la presencia de los MP en estudio. En el ACP se agregó la variable altitud (en m), la cual se obtuvo del Modelo Digital de Elevación (MDE) de México (INEGI, 2013b).

Mediante análisis visual de histogramas y pruebas Shapiro se determinó que los datos para $\mathrm{Hg}$ y Fe $(\mathrm{p}<0.005)$ no siguieron la distribución normal, por lo que se procedió a realizar pruebas no paramétricas. Para contrastar las concentraciones de $\mathrm{Cd}$ y $\mathrm{Cu}$ en las zonas A, B y C se aplicó la prueba de análisis de varianza (ANOVA) así como la prueba no paramétrica Kruskal-Wallis para el $\mathrm{Hg}$ y el Fe (Moore, 2005). Se aplicaron Pruebas $t$ de Student para contrastar las concentraciones de $\mathrm{Cd}$ y $\mathrm{Cu}$ por tipo de suelo y posteriormente por sustrato, mientras que para el $\mathrm{Hg}$ y el Fe se aplicó la prueba no paramétrica Man-Whitney (Moore, 2005).

Las distancias entre cada sitio dentro del canal reportadas en la Tabla 2 se obtuvieron con la herramienta "Regla" de ArcMAp. Se generaron mapas para mostrar patrones de distribución de cada elemento, representando la magnitud de concentración con círculos. Las direcciones de flujo de agua presentadas en los mapas fueron generadas con la herramienta "Dirección de Flujo" a partir del MDE, usando el programa ArcMAp v.10.2 (ArcGIS).

\section{RESULTADOS Y DISCUSIÓN}

\section{Parámetros fisicoquímicos}

Los resultados de los parámetros fisicoquímicos determinados para suelos se presentan en la Tabla 1 y los parámetros estadísticos básicos en la Tabla 3. El contenido de MO tuvo en promedio $0.80 \pm 0.12 \%$ con 
Tabla 3. Parámetros estadísticos básicos de metales pesados y parámetros fisicoquímicos en suelos.

\begin{tabular}{lcccccc}
\hline Parámetro & $\begin{array}{c}\text { Número de } \\
\text { observaciones }\end{array}$ & Promedio & Mínimo & Máximo & DE & \% CV \\
\hline $\mathrm{Hg}\left(\mu \mathrm{g} \cdot \mathrm{kg}^{-1}\right)$ & 18 & 24.07 & 3.80 & 160.00 & 37.49 & 155.84 \\
$\mathrm{Cd}\left(\mathrm{mg} \cdot \mathrm{kg}^{-1}\right)$ & 18 & 0.55 & 0.05 & 1.00 & 0.33 & 60.51 \\
$\mathrm{Cu}\left(\mathrm{mg} \cdot \mathrm{kg}^{-1}\right)$ & 18 & 10.56 & 0.90 & 23.84 & 5.52 & 52.22 \\
$\mathrm{Fe}\left(\mathrm{mg} \cdot \mathrm{kg}^{-1}\right)$ & 18 & $25,334.13$ & $8,786.50$ & $53,624.10$ & $15,341.79$ & 60.55 \\
$\mathrm{MO}(\%)$ & 18 & 0.80 & 0.52 & 1.05 & 0.12 & 15.14 \\
$\mathrm{pH}$ & 18 & 6.53 & 5.23 & 8.09 & 0.89 & 13.72 \\
$\mathrm{CIC}\left(\mathrm{Cmol} \cdot \mathrm{kg}^{-1}\right)$ & 18 & 44.46 & 43.20 & 45.00 & 0.49 & 1.11 \\
\hline
\end{tabular}

$\mathrm{DE}=$ Desviación Estándar; $\mathrm{CV}=$ Coeficiente de variación.

rango de $0.52-1.05 \%$, un coeficiente de variación (CV) de $15.14 \%$, lo que indica que en general los suelos de la zona de estudio tienen bajo contenido de MO. Por otra parte, presentan un $\mathrm{pH}$ moderadamente ácido a medianamente alcalino, de acuerdo a la clasificación establecida por SEMARNAT (2002), con un valor promedio de $6.53 \pm 0.89$, un rango de $5.23-8.09 \mathrm{y}$ un CV de $13.72 \%$. La CIC en promedio fue de $44.46 \pm 0.49 \mathrm{Cmol} \cdot \mathrm{kg}^{-1}$ con rango de $43.20-45.00 \mathrm{Cmol} \cdot \mathrm{kg}^{-1} \mathrm{y}$ un $\mathrm{CV}$ de $1.11 \%$. Esto indica que los suelos analizados tienen una CIC muy alta de acuerdo a los valores establecidos por SEMARNAT (2002), que puede reflejarse en una alta retención de iones con carga positiva, entre ellos los MP.

Los resultados de las variables fisicoquímicas para los sedimentos se presentan en la Tabla 2 y los estadísticos básicos en la Tabla 4 . El contenido de $\mathrm{MO}$ en general fue bajo (SEMARNAT, 2002) con un promedio de $0.54 \pm 0.07 \%$, un rango de $0.45-0.66 \%$ y un CV de $12.63 \%$; el pH fue generalmente alcalino (SEMARNAT, 2002) con un promedio de $7.9 \pm 0.20$, un rango de $7.57-8.11$ y CV de $2.52 \%$.

\section{Concentraciones de MP en suelos y sedimentos}

Con el fin de unificar unidades para realizar comparaciones, los datos de $\mathrm{Hg}$ se convirtieron en $\mathrm{mg} \cdot \mathrm{kg}^{-1}$, mientras que en las tablas, gráficos y figuras referidas, las concentraciones se presentan en el orden de $\mu \mathrm{g} \cdot \mathrm{kg}^{-1}$. En la Tabla 1 se presentan las concentraciones de MP detectadas en suelo; en orden descendente fueron $\mathrm{Fe}>\mathrm{Cu}>$ $\mathrm{Cd}>\mathrm{Hg}$ con valores promedio de $25,334.13 \pm 15,341.79 \mathrm{mg} \cdot \mathrm{kg}^{-1}$, $10.56 \pm 5.52 \mathrm{mg} \cdot \mathrm{kg}^{-1}, 0.55 \pm 0.33 \mathrm{mg} \cdot \mathrm{kg}^{-1}$ y $0.02 \pm 0.03 \mathrm{mg} \cdot \mathrm{kg}^{-1}$, respectivamente. Los rangos para los mismos elementos fueron: $8,786.50$ $53,624.10 \mathrm{mg} \cdot \mathrm{kg}^{-1}, 0.90-23.84 \mathrm{mg} \cdot \mathrm{kg}^{-1}, 0.05-1.00 \mathrm{mg} \cdot \mathrm{kg}^{-1} \mathrm{y} 0.03-0.16$ $\mathrm{mg} \cdot \mathrm{kg}^{-1}$, respectivamente. Los valores de CV fueron $60.55 \%, 52.22 \%$, $60.51 \%$ y $155.84 \%$, respectivamente (Tabla 3), siendo el Fe el elemento más abundante, mientras que el $\mathrm{Hg}$ es el de menor concentra- ción. Las concentraciones promedio de $\mathrm{Cd}, \mathrm{Cu}$ y Fe $\left(646.89 \mathrm{mg} \cdot \mathrm{kg}^{-1}\right.$, $10.64 \mathrm{mg} \cdot \mathrm{kg}^{-1}$ y $23,004.83 \mathrm{mg} \cdot \mathrm{kg}^{-1}$, respectivamente) en suelos de tipo vertisol (Tabla 5) resultaron mayores a las encontradas por De la CruzPons et al. (2012) en suelos cultivados con caña de azúcar en Tabasco.

Las concentraciones de MP en sedimento se muestran en la Tabla 2. Las concentraciones en orden descendente fueron $\mathrm{Fe}>\mathrm{Cu}$ $>\mathrm{Cd}>\mathrm{Hg}$ con valores promedio de 19,952.68 $\pm 10,379.02 \mathrm{mg} \cdot \mathrm{kg}^{-1}$, $8.00 \pm 5.52 \mathrm{mg} \cdot \mathrm{kg}^{-1}, 0.54 \pm 0.16 \mathrm{mg} \cdot \mathrm{kg}^{-1} \mathrm{y} 0.06 \pm 0.03 \mathrm{mg} \cdot \mathrm{kg}^{-1}$, respectivamente. Los rangos fueron $11,064.19-46,261.19 \mathrm{mg} \cdot \mathrm{kg}^{-1}, 2.05-19.82 \mathrm{mg} \cdot \mathrm{kg}^{-1}$, $0.30-0.75 \mathrm{mg} \cdot \mathrm{kg}^{-1}$ y $0.005-0.12 \mathrm{mg} \cdot \mathrm{kg}^{-1}$, respectivamente. Las proporciones en el CV fueron de $52.02 \%, 69.08 \%$, $30.18 \%$ y $55.90 \%$, respectivamente (Tabla 4). Al igual que en los suelos, el elemento más abundante es el $\mathrm{Fe}$, mientras que el $\mathrm{Hg}$ fue el de menor concentración.

Ungureanu et al. (2016) sugirieron que los CV de las concentraciones de MP que dominan en la naturaleza son relativamente bajos, mientras que los CV de las concentraciones de los elementos afectados por actividades antropogénicas son bastante altos. En este estudio los $\mathrm{CV}$ de los MP fueron de $52.22 \%$ de Cu hasta $155.84 \%$ de Hg. Por lo tanto, de acuerdo con estos autores, podemos inferir que las concentraciones de MP detectadas en los suelos han sido influenciadas por fuentes de origen antropogénico.

De acuerdo con Segura-Muñoz et al., (2005), los MP en suelos provienen de diferentes fuentes, que van desde su origen natural (dependiendo del área geográfica) hasta las antropogénicas (asociadas principalmente a actividades como la agricultura, minería, procesos industriales, etc.) y de diferentes vías, como el desgaste del material parental, el vertido de aguas residuales sin tratamiento, la aplicación de biosólidos como abono, el uso de fertilizantes inorgánicos y plaguicidas, el depósito atmosférico, etc. (Wang et al., 2015). Los asentamientos humanos cercanos al área de estudio no cuentan con un relleno sanitario, por lo tanto, los residuos domiciliarios son depositados en

Tabla 4. Parámetros estadísticos básicos de metales pesados y parámetros fisicoquímicos en sedimentos.

\begin{tabular}{lcrrrrr}
\hline Parámetro & $\begin{array}{c}\text { Número de } \\
\text { observaciones }\end{array}$ & Promedio & Mínimo & Máximo & DE & \% CV \\
\hline $\mathrm{Hg}\left(\mu \mathrm{g} \cdot \mathrm{kg}^{-1}\right)$ & 18 & 63.98 & 5.96 & 127.26 & 35.77 & 55.90 \\
$\mathrm{Cd}\left(\mathrm{mg} \cdot \mathrm{kg}^{-1}\right)$ & 18 & 0.54 & 0.30 & 0.75 & 0.16 & 30.18 \\
$\mathrm{Cu}\left(\mathrm{mg} \cdot \mathrm{kg}^{-1}\right)$ & 18 & 8.00 & 2.05 & 19.82 & 5.52 & 69.08 \\
$\mathrm{Fe}\left(\mathrm{mg} \cdot \mathrm{kg}^{-1}\right)$ & 18 & $19,952.68$ & $11,064.19$ & $46,261.53$ & $10,379.02$ & 52.02 \\
$\mathrm{MO}(\%)$ & 18 & 0.54 & 0.45 & 0.66 & 0.07 & 12.63 \\
$\mathrm{pH}$ & 18 & 7.90 & 7.57 & 8.11 & 0.20 & 2.52 \\
\hline
\end{tabular}

$\mathrm{DE}=$ Desviación Estándar; $\mathrm{CV}=$ Coeficiente de variación. 
Tabla 5. Concentraciones promedio de metales pesados y parámetros fisicoquímicos por tipo de suelo.

\begin{tabular}{lcc}
\hline Parámetro & Gleysol & Vertisol \\
\hline $\mathrm{Hg}\left(\mu \mathrm{g} \cdot \mathrm{kg}^{-1}\right)$ & $15.72 \pm 17.40$ & $32.41 \pm 50.2715$ \\
$\mathrm{Cd}\left(\mathrm{mg} \cdot \mathrm{kg}^{-1}\right)$ & $0.67 \pm 0.31$ & $0.43 \pm 0.33$ \\
$\mathrm{Cu}\left(\mathrm{mg} \cdot \mathrm{kg}^{-1}\right)$ & $10.50 \pm 7.01$ & $10.64 \pm 3.94$ \\
$\mathrm{Fe}\left(\mathrm{mg} \cdot \mathrm{kg}^{-1}\right)$ & $27,663.44 \pm 16,795.06$ & $23,004.83 \pm 14,348.53$ \\
$\mathrm{CIC}\left(\mathrm{Cmol} \cdot \mathrm{kg}^{-1}\right)$ & $44.29 \pm 0.65$ & $44.63 \pm 0.17$ \\
$\mathrm{MO}(\%)$ & $0.78 \pm 0.13$ & $0.82 \pm 0.12$ \\
$\mathrm{pH}$ & $5.88 \pm 0.38$ & $7.19 \pm 0.77$ \\
$\mathrm{n}$ & 9 & 9 \\
\hline
\end{tabular}

áreas alejadas de los centros de población, pero cercanas a las zonas de cultivo. En estas áreas de depósito gran parte de los residuos son incinerados, representando así una fuente potencial de contaminación por MP vía depósito atmosférico; por otro lado, los gases de las chimeneas del ingenio azucarero también representan una fuente de contaminación. Otra fuente importante de estos elementos es el uso de agroquímicos. En el cultivo de caña de azúcar se ha utilizado por años la fertilización a base de mezclas de nitrógeno $(\mathrm{N})$, potasio $(\mathrm{K})$ y fósforo $(\mathrm{P})$ sin considerar los requerimientos de la variedad de la planta o el tipo de suelo (Salgado-García et al., 2000; Palma-López et al., 2002). En particular, en la zona cañera de Quintana Roo la cantidad de fertilizantes vertidos de 1979 a 2016 asciende a un total aproximado de $86,200 \mathrm{~kg} / \mathrm{ha}$. Las fórmulas a base de $\mathrm{N}, \mathrm{K}$ y P son las más usadas con un valor aproximado de $79,150 \mathrm{~kg} / \mathrm{ha}$; el sulfato de amonio y urea son los de menor uso, con un valor aproximado de 2,250 y 4,800 kg/ha, respectivamente (Iuit-Jiménez, 2017). La aplicación, en ocasiones excesiva, de agroquímicos ha ocasionado el incremento de las concentraciones de MP en el suelo, ya que contienen trazas de estos elementos. Esto fue comprobado por Gimeno-García et al. (1995) al analizar diversos agroquímicos y determinar que los fertilizantes a base de fosfato contenían los niveles más altos de Cd, Co, Cu y Zn. Además Gimeno-García et al. (1995) encontraron que entre los plaguicidas que estudiaron, los herbicidas Saturn-G y Ordram contenían las concentraciones más altas de $\mathrm{Fe}, \mathrm{Mn}, \mathrm{Zn}, \mathrm{Pb}$ y Ni. WenHuang y Yun-Jin (2008) hicieron un estudio sobre el estado de los MP en suelos agrícolas de la provincia de Huabei, China, afectada por diferentes patrones de uso de suelo; concluyeron que el uso excesivo de fertilizantes a base de fósforo puede conducir a la acumulación de $\mathrm{Cd}, \mathrm{Cu}, \mathrm{Zn}$ y otros MP a largo plazo. Atafar et al. (2010) obtuvieron resultados similares en un estudio sobre el efecto de fertilizantes en suelos de la provincia de Kermanshash, Irán, en el cual reportaron que los fertilizantes compuestos y triple super-fosfato contenían la mayor concentración de $\mathrm{Cd}$; para el $\mathrm{Pb}$ y As la concentración máxima se encontró en un fertilizante a base de sulfato de zinc, reportando que las concentraciones de $\mathrm{Cd}, \mathrm{Pb}$ y As aumentaron en los suelos después de la aplicación de estos fertilizantes. Cheraghi et al. (2012) coincidieron en que los fertilizantes a base de fosfato son fuente importante de MP ya que la materia prima de estos productos se obtiene de depósitos de fosforita que, en general, contienen altas concentraciones de $\mathrm{Cu}, \mathrm{Cd}$, $\mathrm{Ni}, \mathrm{Zn}, \mathrm{Co}, \mathrm{Pb}$ y $\mathrm{Mn}$. En el mismo sentido, es importante mencionar que algunos productos comerciales recomendados para el cultivo de caña reportan en su ficha técnica que contienen los siguientes MP: $\mathrm{Al}, \mathrm{Cd}, \mathrm{Cu}, \mathrm{Fe}, \mathrm{Mg}, \mathrm{Mn}, \mathrm{Ni}, \mathrm{Pb}, \mathrm{Zn}$ y As (Palau-Bioquim, s.f.; Bioterra Internacional, s.f.; VELSIMEX, 2009; BEB, 2015; CICOPLAFEST, SESA, SAGARPA, SEMARNAT, 2004; El Vergel de Occidente S.A. de C.V., s.f.). En ninguna de las fichas técnicas consultadas se reportan concentraciones de $\mathrm{Hg}$; sin embargo, se conoce que los fungicidas usados para controlar las enfermedades de la caña de azúcar son elaborados a base de compuestos orgánicos de $\mathrm{Hg}$ (Yadav et al., 2010).

Las concentraciones altas de Fe en este estudio, se deben a que se realizó en un área donde el suelo predominante es el tipo gleysol, que se caracteriza por contener segregados de compuestos de este elemento, generalmente en los primeros $50 \mathrm{~cm}$ (FAO, 2014). Sin embargo, estas concentraciones también han sido influenciadas por la actividad agrícola, ya que al labrar los suelos, la estructura de éstos es modificada, propiciando la redistribución de Fe y otros componentes del suelo por medio de la erosión provocada por la escorrentía en la época de lluvias (FAO, 2017).

\section{Relación entre MP y parámetros fisicoquímicos}

Con el objetivo de identificar las variables que describan la distribución de los MP en el área de estudio, se realizaron correlaciones de Pearson entre los MP y los parámetros fisicoquímicos de cada matriz (Tablas 6 y 7 ). Se ha reportado que en el suelo la CIC y la MO juegan un papel importante en la retención de los MP y el pH en la disposición de éstos, y por ello, concentraciones altas de MP se relacionan con estos parámetros (De Matos et al., 2001; Dube et al., 2001; Clemente et al., 2008; Violante et al., 2010; Kennou et al., 2015; Chandrasekaran y Ravisankar, 2015). En cultivos de caña de azúcar se recomiendan productos como Algaenzims ${ }^{\oplus}$ y Amikrone ${ }^{\circledR}$ para mejorar las propiedades físicas, químicas y biológicas del suelo y ayudar en la retención de los nutrientes. Sin embargo, estos agroquímicos no son selectivos y también influyen en los MP (Palau-Bioquim, s.f.; BEB, 2015). En este estudio no se obtuvieron relaciones significativas entre las concentraciones de MP y los parámetros fisicoquímicos, con excepción de una correlación negativa entre el $\mathrm{Cd}$ y $\mathrm{pH}$ $(\mathrm{r}=-0.6, \mathrm{p}<0.01)$ y positiva entre el $\mathrm{Cu}$ y Fe $(\mathrm{r}=0.63, \mathrm{p}<0.01)$ en suelos (Tabla 6). Estas relaciones indican que en el área de estudio, el Cd se está reteniendo más en suelos ácidos y que las concentraciones de $\mathrm{Cu}$, posiblemente, están siendo retenidas por los óxidos e hidróxidos de $\mathrm{Fe}$ del suelo (Acevedo-Sandoval, et.al., 2004), compuestos conocidos por asociarse con la retención de MP (Tessier et al., 1979). En sedimentos (Tabla 7) se obtuvo una relación positiva entre las concentraciones de $\mathrm{Hg}$ y $\mathrm{Cu}(\mathrm{r}=0.69, \mathrm{p}<0.05)$; esta relación indica que estos elementos están siguiendo un patrón de distribución similar en los sedimentos superficiales del canal.

\section{Distribución de MP en suelos y sedimentos}

Para identificar los patrones de distribución de los MP se consideró la orografía; las altitudes de cada sitio de muestreo se obtuvieron del mapa elaborado con el MDE. Se analizaron las concentraciones de cada elemento por zona; los análisis estadísticos realizados a escala del área de estudio arrojaron que únicamente el Cd presenta diferencias significativas entre las zonas $(\mathrm{p}<0.05)$. Pero si se observa el mapa de la distribución de cada elemento (Figura 2) y las gráficas de altitud $v s$. concentración de MP encontramos que aparentemente en la zona A

Tabla 6. Correlaciones entre metales pesados y parámetros fisicoquímicos en suelos.

\begin{tabular}{llrlclll}
\hline Parámetro & $\mathbf{H g}$ & $\mathbf{C d}$ & $\mathbf{C u}$ & $\mathbf{F e}$ & $\mathbf{p H}$ & $\mathbf{M O}$ & $\mathbf{C I C}$ \\
\hline $\mathrm{Hg}$ & 1 & & & & & & \\
$\mathrm{Cd}$ & 0.30 & 1 & & & & & \\
$\mathrm{Cu}$ & 0.08 & 0.23 & 1 & & & & \\
$\mathrm{Fe}$ & 0.39 & 0.39 & 0.63 & 1 & & & \\
$\mathrm{pH}$ & -0.08 & -0.60 & 0.14 & -0.15 & 1 & & \\
$\mathrm{MO}$ & 0.09 & 0.13 & 0.41 & 0.42 & 0.34 & 1 & \\
$\mathrm{CIC}$ & 0.21 & -0.26 & -0.51 & -0.35 & 0.11 & -0.38 & 1 \\
\hline
\end{tabular}


Tabla 7. Correlaciones entre metales pesados y parámetros fisicoquímicos en sedimentos.

\begin{tabular}{lllllll}
\hline Parámetro & $\mathbf{H g}$ & $\mathbf{C d}$ & $\mathbf{C u}$ & $\mathbf{F e}$ & $\mathbf{p H}$ & MO \\
\hline $\mathrm{Hg}$ & 1 & & & & & \\
$\mathrm{Cd}$ & 0.45 & 1 & & & & \\
$\mathrm{Cu}$ & $\mathbf{0 . 6 9}$ & 0.37 & 1 & & & \\
$\mathrm{Fe}$ & 0.41 & 0.54 & 0.55 & 1 & & \\
$\mathrm{pH}$ & 0.49 & -0.28 & 0.11 & 0.17 & 1 & \\
$\mathrm{MO}$ & -0.08 & -0.05 & 0.47 & -0.04 & -0.37 & 1 \\
\hline
\end{tabular}

(Figura 3) la concentración más alta de cada elemento se encuentra en el canal. En la zona B (Figura 4) se observa que las concentraciones más elevadas se hayan en el noreste de ésta, con excepción de los valores para Cd que se perciben con una distribución más homogénea en toda la zona. Además, esta zona aparenta contener las mayores concentra- ciones de $\mathrm{Hg}, \mathrm{Cd}, \mathrm{Cu}$ y Fe. Esto se debe a que esta zona presenta las altitudes más bajas ( $30 \mathrm{~m}$ ), lo cual ocasiona que el flujo de agua sea más lento, propiciando el depósito de las partículas de suelos y los residuos de agroquímicos (que contienen MP) y a su vez su acumulación. En la zona C (Figura 5) se observan valores similares de concentración en ambos lados del canal y los valores más bajos dentro del mismo. Este patrón se aprecia para todos los elementos. Si se observan únicamente los valores de concentración de cada metal encontrado en los sedimentos del canal (Figura 6), se aprecia que el $\mathrm{Hg}$ sigue un patrón de dispersión a lo largo de éste, mientras que el $\mathrm{Cu}, \mathrm{Cd}$ y Fe se acumulan en la parte media del cauce (zona B) donde la altitud es homogénea $(30 \mathrm{~m})$.

Las concentraciones de $\mathrm{Hg}$ y $\mathrm{Fe}$ se distribuyen de manera uniforme en ambos tipos de suelo, gleysol y vertisol ( $p>0.05)$, mientras que las concentraciones de $\mathrm{Cd}$ y Cu resultaron ser mayores $(\mathrm{p}<0.05)$ en los suelos de tipo gleysol. A pesar de que las correlaciones de Pearson arrojaron alta correlación entre $\mathrm{Cu}$ y Fe, al comparar por tipo de suelo, estos elementos no se asocian.
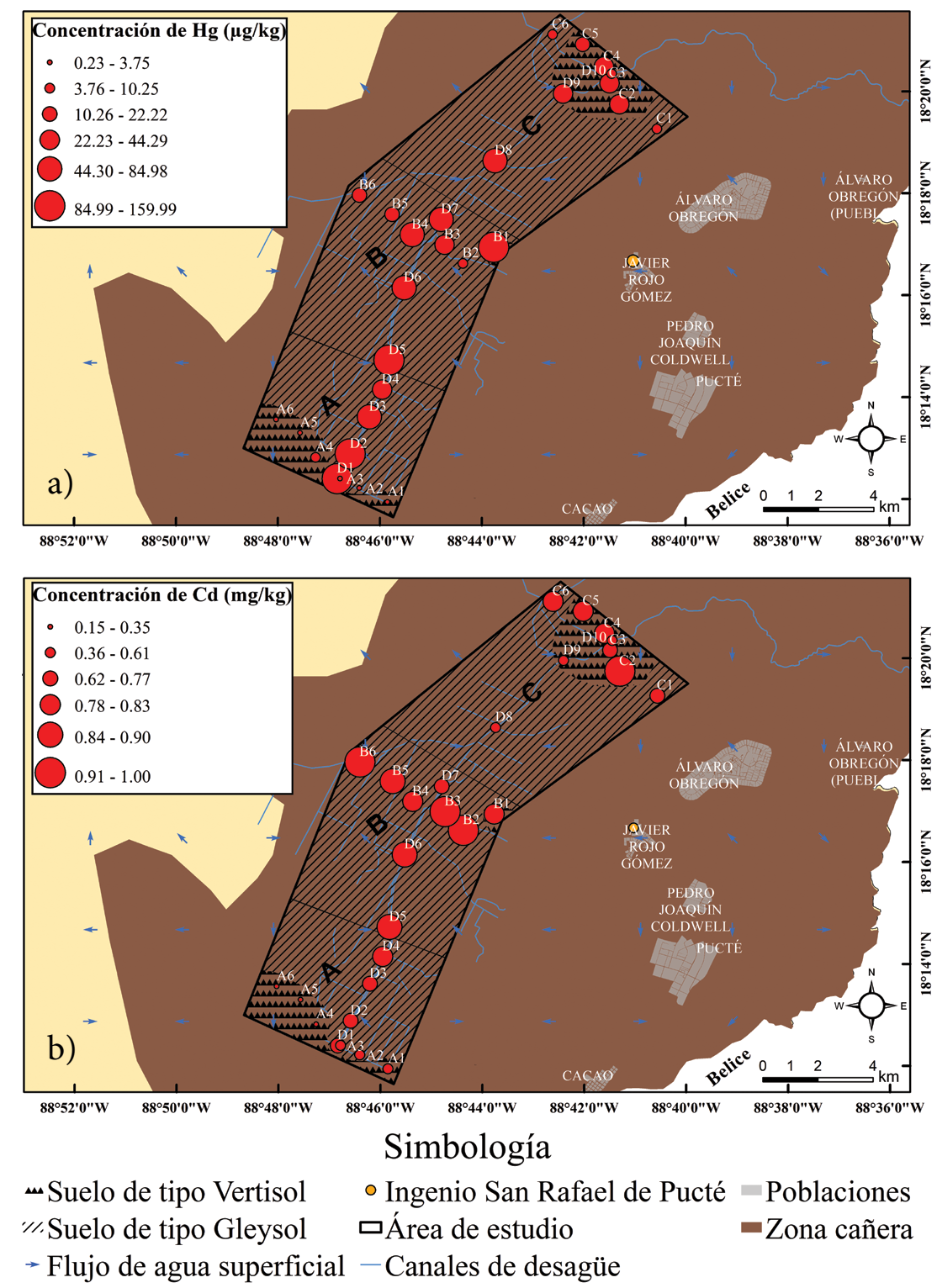

Figura 2. Mapas de distribución de metales pesados. a) distribución de mercurio; b) distribución de cadmio. El Datum es WGS 1984. Continúa. 


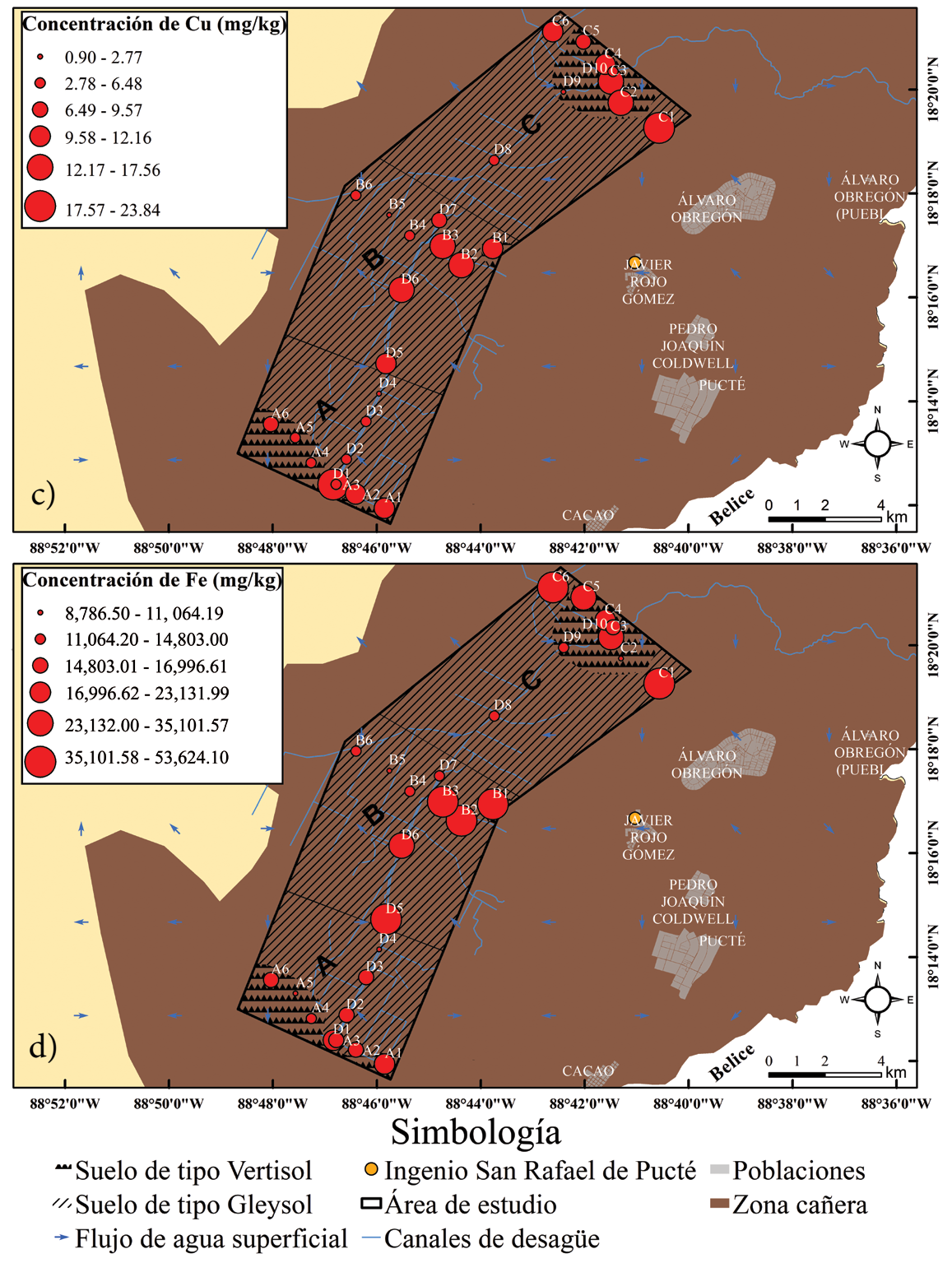

Figura 2 (continuación). Mapas de distribución de Metales Pesados. c) Distribución de cobre; d) Distribución de hierro. El Datum es WGS 1984.

Al contrastar las concentraciones de cada MP con la matriz ambiental, se aprecia que las concentraciones más altas de $\mathrm{Hg}$ se encuentran en los sedimentos ( $\mathrm{p}<0.05)$; el Cd y el Cu quedan retenidos en los suelos $(\mathrm{p}<0.05)$ y el Fe se distribuye de manera homogénea en ambas matrices $(\mathrm{p}>0.05)$.

En el ACP se agregó la variable altitud para ambas matrices (Figura 7), la cual aparentemente no se asocia con las concentraciones de MP; esto coincide con los resultados obtenidos por las correlaciones de Pearson. Sin embargo, en los grupos generados por el AC, los parámetros fisicoquímicos muestran cierta relación con los MP (Figura 8). En suelos, el Grupo 1 incluye los sitios con concentraciones más bajas de MP, los valores más altos de CIC, valores intermedios de $\mathrm{pH}$ y los porcentajes de MO. El Grupo 2 incluye las concentraciones intermedias de los MP, los valores más altos de $\mathrm{pH}$ y $\mathrm{MO}$ e intermedios de CIC. En el Grupo 3 se encuentran los sitios con concentraciones más altas de MP, valores más bajos de pH y CIC, así como los valores intermedios de $\mathrm{MO}$. El tipo de suelo también es un factor representativo de los grupos: los suelos de tipo vertisol representan a los grupos con las menores concentraciones de MP (Grupos 1 y 2), y los suelos de tipo gleysol al Grupo 3, el cual corresponde a los sitios con mayor concentración de MP. Ambos tipos de suelo son influenciados por el agua. Los de tipo gleysol son suelos que se saturan con agua freática durante largos periodos, lo cual les proporciona una baja conductividad hidráulica. Los suelos de tipo vertisol cuentan con una estructura pesada y predominio de minerales de arcillas expandibles, lo cual resulta en grietas profundas en la temporada de sequía. Este tipo de suelo cuenta con infiltración más rápida en comparación con los de tipo gleysol (FAO, 2014). En ambos tipos de suelo existe arrastre de partículas: en los de tipo gleysol, el transporte es superficial debido a que estos suelos se saturan, mientras que en los de tipo vertisol el movimiento es a través del perfil del suelo; estas características explicarían la retención de los MP en la superficie de los suelos de tipo gleysol.

Los sitios localizados dentro del canal (sedimentos) también fueron categorizados en tres grupos (Figura 9). El Grupo 1 se caracteriza por tener las concentraciones más altas de $\mathrm{Cd}$ y Fe, concentraciones 

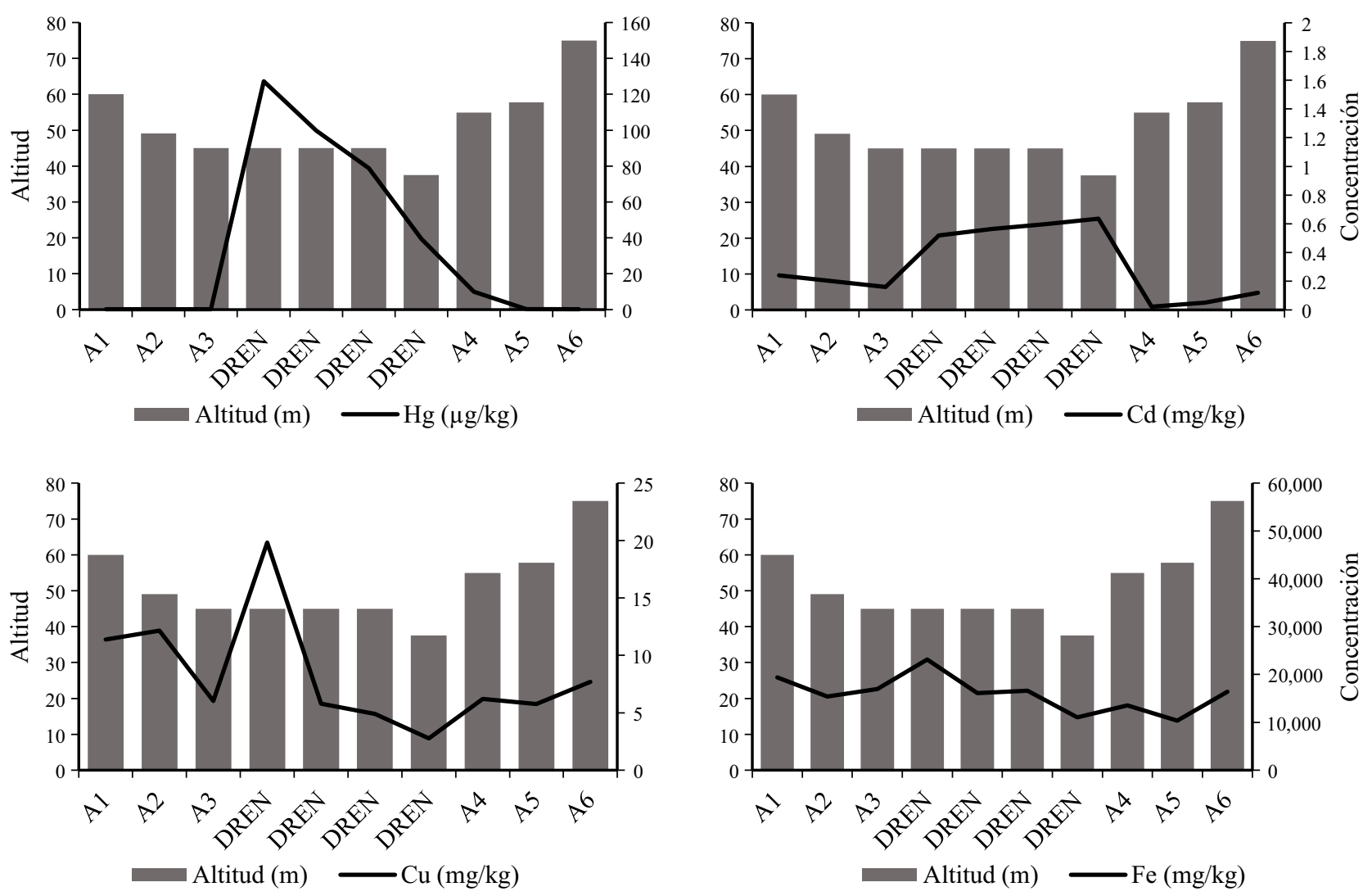

Figura 3. Gráficas de altitud y concentraciones de Metales Pesados de la zona A.

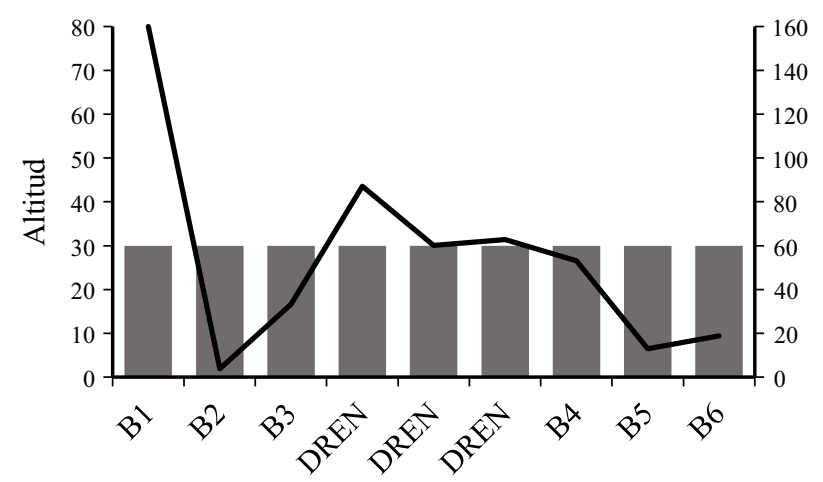

Altitud (m) —Hg $(\mu \mathrm{g} / \mathrm{kg})$
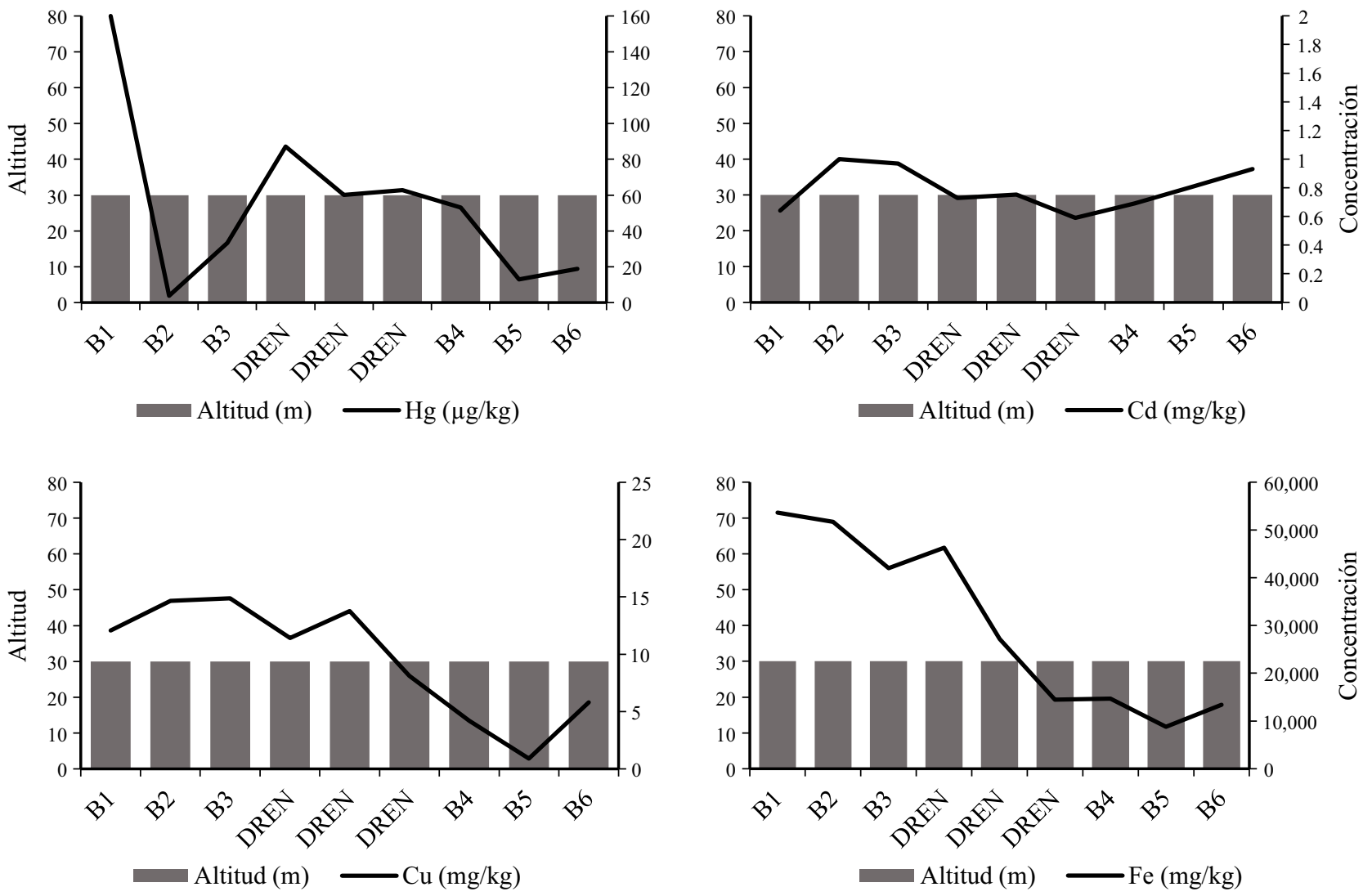

Figura 4. Gráficas de altitud y concentraciones de metales pesados de la zona B. 

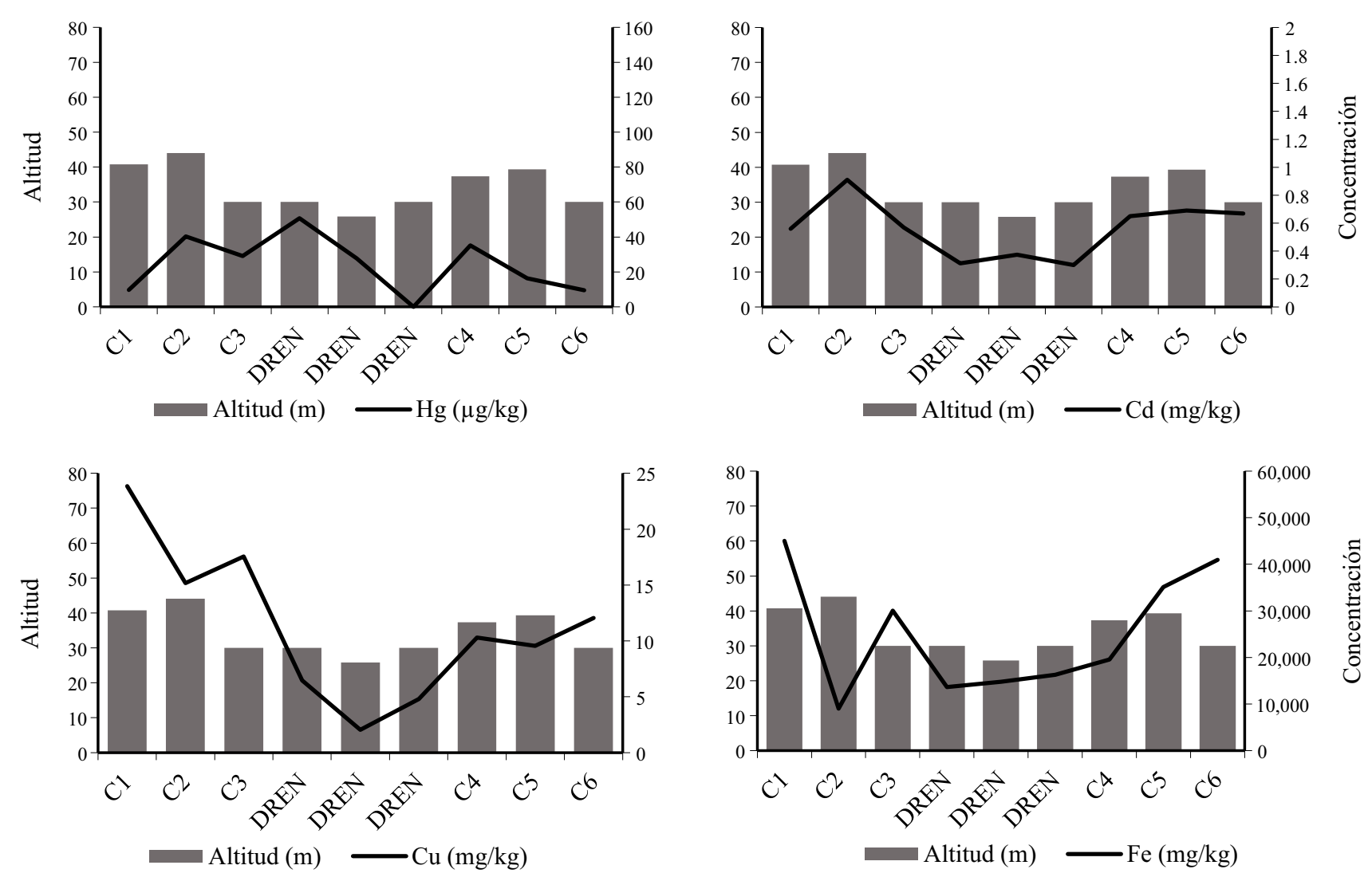

Figura 5. Gráficas de altitud y concentraciones de metales pesados de la zona C.
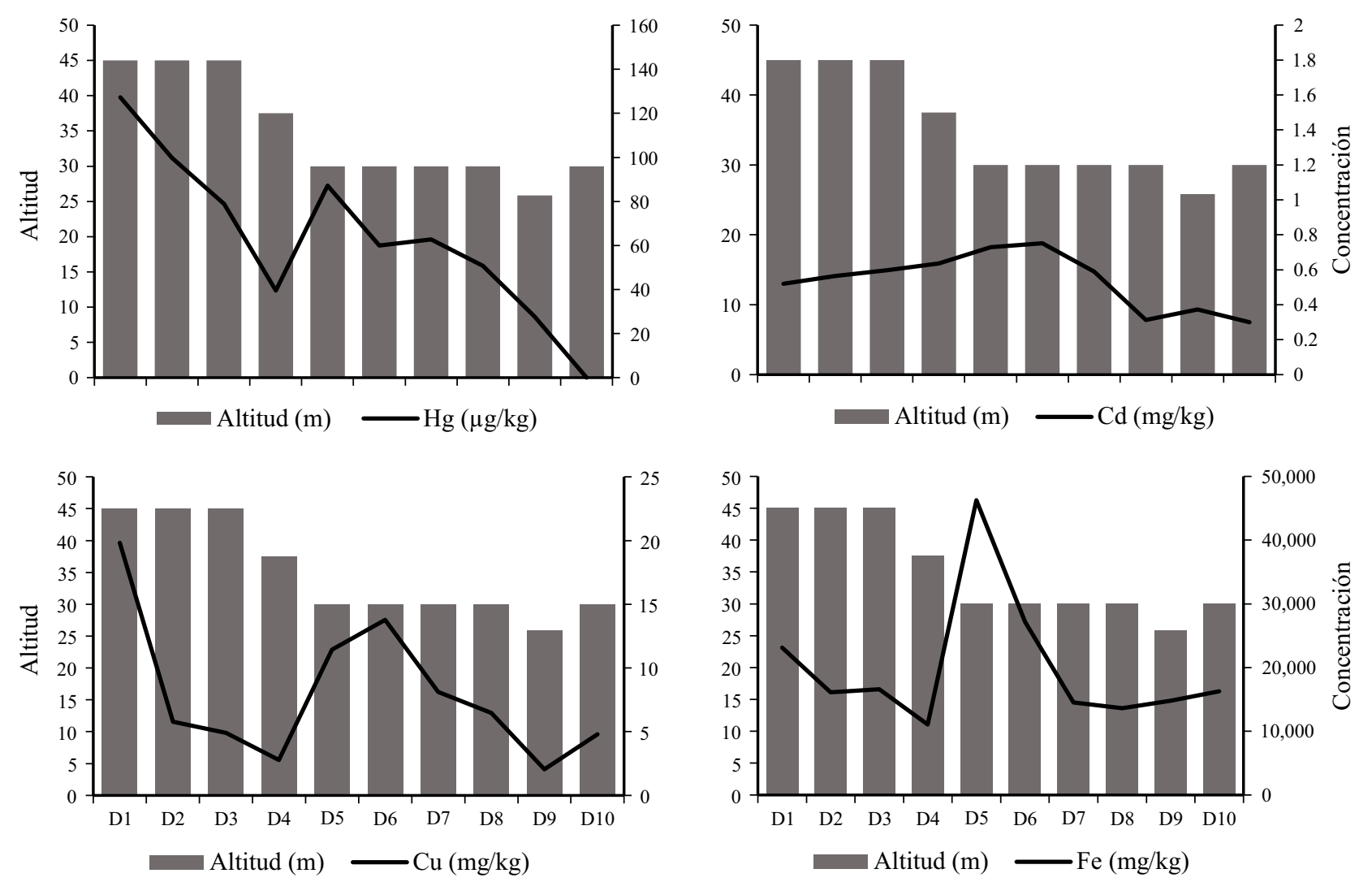

Figura 6. Gráficas de altitud y concentraciones de metales pesados en las muestras de sedimentos. 

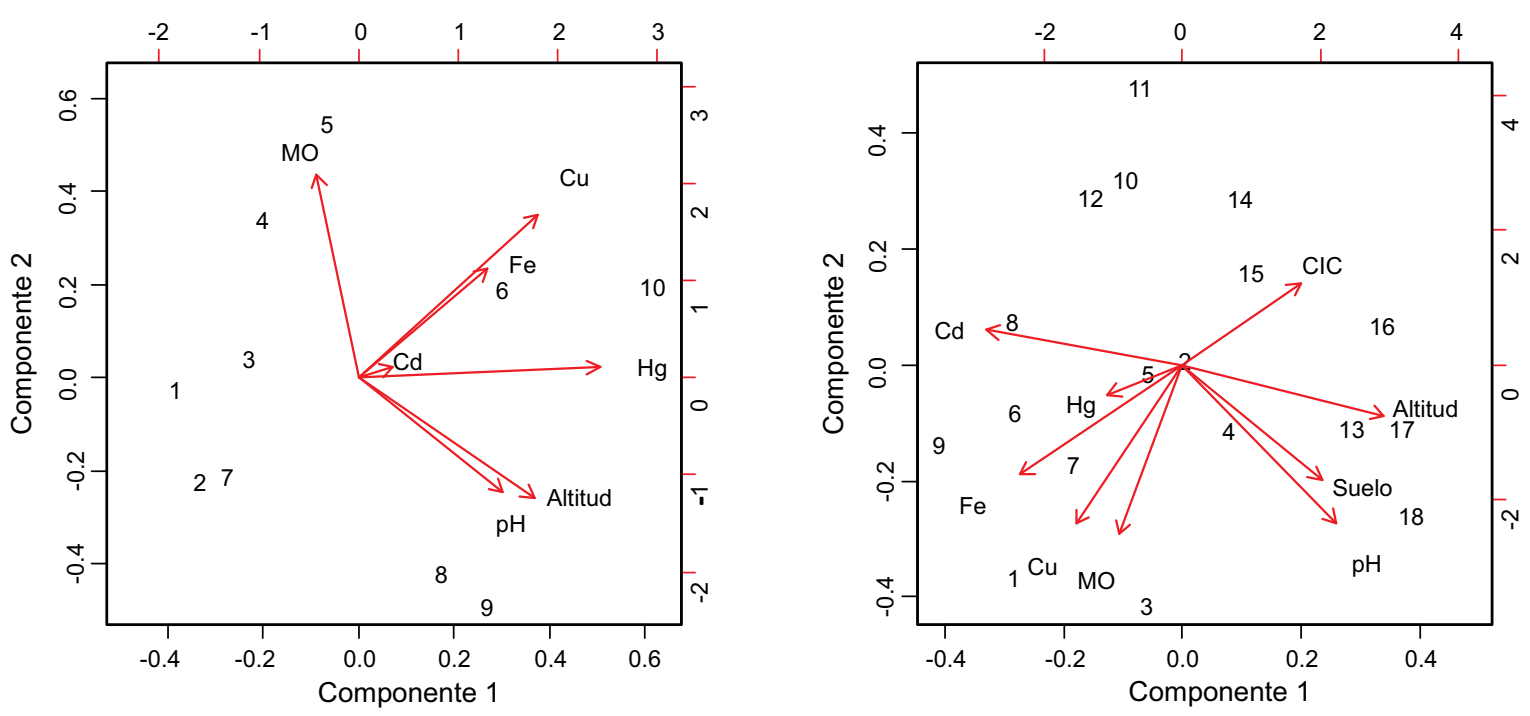

Figura 7. Gráficas de análisis de componentes principales de las muestras en sedimentos (izquierda) y suelos (derecha).

intermedias de $\mathrm{Hg}$ y $\mathrm{Cu}$, los valores más altos de $\mathrm{pH}$ y los porcentajes más bajos de MO. Por otro lado, el Grupo 2 incluye los sitios con concentraciones más altas de $\mathrm{Hg}$ y $\mathrm{Cu}$, concentraciones intermedias de $\mathrm{Cd}$ y $\mathrm{Fe}$, los valores más bajos de $\mathrm{pH}$ y los porcentajes más altos de MO. Por último, en el Grupo 3 se encuentran los sitios con las concentraciones más bajas de los MP y valores intermedios de $\mathrm{pH}$ y MO. El AC ratifica los resultados del comportamiento de los MP en los sedimentos del canal: el Grupo 1 tiene las concentraciones más altas del Fe y Cd y concentraciones intermedias de $\mathrm{Hg}$ y Cu; también el Grupo 1 tiene los sitios con menor altitud y distancias intermedias respecto al inicio del canal. El Grupo 2 incluye las concentraciones más altas de $\mathrm{Hg}$ y Cu , así como concentraciones intermedias del Cd y Fe; además, tiene los sitios de mayor altitud y más cercanos al inicio del canal. Por último, el Grupo 3 muestra las concentraciones más bajas de todos los MP; este grupo tiene los sitios más alejados del inicio del canal, con altitudes intermedias.

Durante la época de lluvias se ha observado que en la zona cañera aumenta considerablemente el caudal. A causa de esto se considera que, en general, el factor que determinaría la distribución de los MP

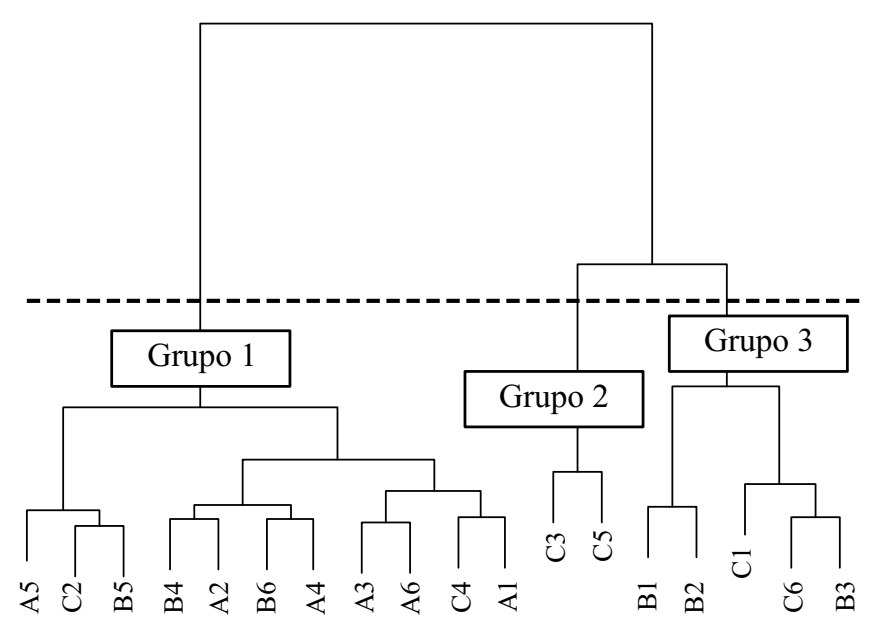

Figura 8. Dendrograma de las muestras de suelos. en el área de estudio es el arrastre de partículas del suelo causado por la escorrentía dirigida hacia las zonas de menor altitud, correspondientes al canal principal de desagüe (la dirección de flujo se puede observar en la Figura 2). La mayor parte del área de estudio corresponde a suelos de tipo gleysol, caracterizados por una baja permeabilidad que causa su saturación en la época de lluvias. Esto propicia el transporte superficial de los MP contenidos en los suelos hacia el dren principal, para posteriormente dispersarse a lo largo del mismo. De esta manera, los MP ingresan al Río Hondo y posteriormente a la bahía de Chetumal. Esto puede relacionarse con el aumento en la turbidez del Río Hondo en la época de lluvias descrito por Álvarez-Legorreta en 2007.

\section{CONCLUSIONES}

Las concentraciones detectadas de $\mathrm{Hg}, \mathrm{Cd}$, Cu parecen provenir de fuentes antropogénicas, mientras que el Fe posiblemente se encuentra de manera natural; sin embargo, la distribución de este elemento podría estar siendo afectada por la actividad agrícola. De acuerdo a los

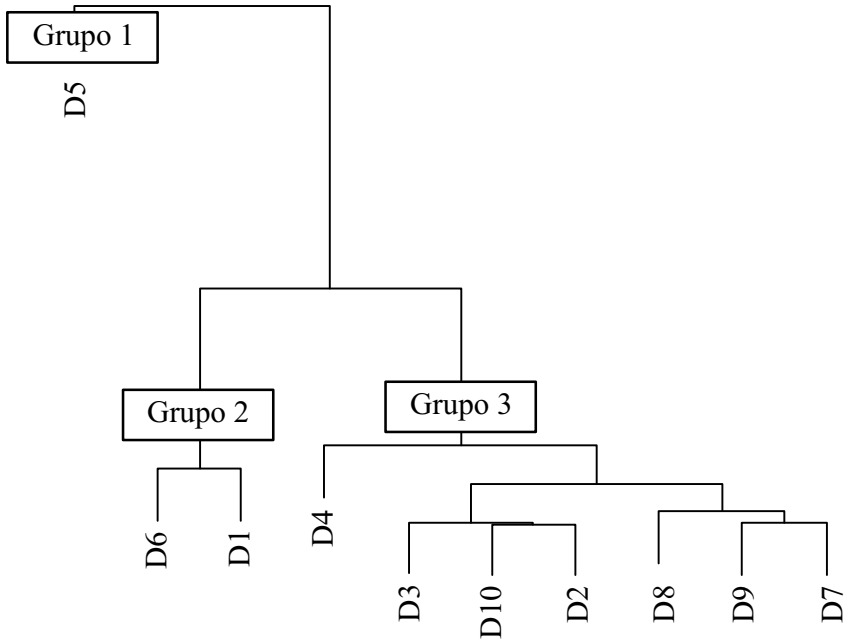

Figura 9. Dendrograma de las muestras de sedimentos. 
análisis estadísticos realizados, no se encontró una asociación entre las concentraciones de los MP y los parámetros fisicoquímicos. El factor determinante en la distribución de los MP es el arrastre de partículas de suelo que los contienen, lo cual ocurre por la escorrentía. De acuerdo al AC, las partículas de suelo con mayor contenido de MP provienen de los suelos de tipo gleysol. Dentro del canal de desagüe, los MP se dispersan de las partes más altas a las más bajas. Adicionalmente, se concluye que el patrón de distribución de los MP comprueba que la actividad agrícola de la zona cañera es una fuente potencial de $\mathrm{Hg}$, $\mathrm{Cd}, \mathrm{Cu}$ y Fe para las aguas superficiales de la cuenca (Río Hondo y bahía de Chetumal) y posiblemente para las aguas subterráneas. Debe tomarse en cuenta que el estudio muestra el patrón de distribución de MP en el tiempo en el que se tomaron las muestras, durante la temporada de secas. Los MP son una fuente potencial para la cuenca, debido a que se observa que las concentraciones de éstos aumentan en los sedimentos del canal principal de desagüe. Además, dentro del canal se observa que las concentraciones de MP se dispersan a través de éste. Esto puede observarse con detalle en las Figuras 3, 4, 5 y 6. También se indica que la zona cañera es una fuente potencial de MP para las aguas subterráneas, debido a que los suelos de esta área son de escaso espesor y se desarrollan sobre un acuífero kárstico, que se caracterizan por una conductividad hidráulica alta, lo cual podría permitir el transporte inmediato de estos contaminantes.

Es necesario realizar un monitoreo de la dispersión de los $\mathrm{MP}$ a través del tiempo, así como estudiar el comportamiento de éstos en el perfil del suelo y en un mayor número de sitios, con el objetivo de obtener información más detallada sobre el comportamiento de los MP en el área de estudio. Se recomienda el uso de fertilizantes considerando los requerimientos de la variedad de la caña de azúcar, así como de las características del tipo de suelo, con el fin de disminuir el uso excesivo de agroquímicos. También se recomienda plantar dentro del canal especies que acumulen biológica y eficientemente los MP, convirtiéndolo en un humedal artificial, para evitar el ingreso de estos elementos a las aguas superficiales de la cuenca. Para tener mayor tiempo de interacción entre agua y planta dentro del canal, se recomienda la construcción de una pequeña presa que regule el flujo de agua en época de estiaje.

\section{AGRADECIMIENTOS}

Al Consejo Nacional de Ciencia y Tecnología (CONACyT) por la beca otorgada para la realización de los estudios de maestría del primer autor. Al Colegio de la Frontera Sur por brindar el equipo y materiales necesarios para la investigación. Al Instituto Nacional de Investigaciones Forestales, Agrícolas y Pecuarias (INIFAP) por su financiamiento para el trabajo de campo. A la Dra. María Aurora Armienta y a un árbitro anónimo por sus comentarios y sugerencias que enriquecieron la presentación de este trabajo.

\section{REFERENCIAS}

Acevedo-Sandoval, O., Ortiz-Hernández, E., Cruz-Sánchez, M., Cruz-Chávez, E., 2004, El papel de los óxidos de hierro en suelos: Terra Latinoamericana, 2(4), 485-497.

Aguilar-Duarte, Y., Bautista, F., Mendoza, E.M., Delgado, C., 2013, Vulnerabilidad y riesgo de contaminación de acuíferos kársticos: Tropical and Subtropical Agroecosystems, 16, 243-263.

Álvarez-Legorreta, T., 2007, Monitoreo de la calidad del agua de la Bahía de Chetumal y el Río Hondo. Informe Técnico. Proyecto CONACYT clave: QROO-2003-CO1-12703, 120 pp.

Atafar, Z., Mesdaghinia, A., Nouri, J., Homaee, M., Yunesian, M.,
Ahmadimoghaddam, M., Mahvi, A.H., 2010, Effect of fertilizer application on soil heavy metal concentration: Environmental Monitoring and Assessment, 160, 83-89.

BEB (Biokrone Excellence in Biotechnology), 2015, Ficha técnica amikrone (en línea). Disponible en: http://www.biokrone.com/fichas/amikrone. pdf; consulta: 22 de febrero de 2016.

Bioterra Internacional S.A. de C.V., s.f., Ficha técnica bio-cañas iniciador (En línea). Disponible en: <http://www.agrolahuerta.com.mx/intranet/mod/ $\mathrm{PLM} / \mathrm{DEAQ} /$ src/productos/8366_28.htm>, acceso libre; consulta, $30 \mathrm{de}$ marzo de 2016.

Buenfil-Rojas, A.M., Álvarez-Legorreta, T., Cedeño-Vázquez, J.R., 2015, Metals and Metallothioneins in Morelet's Crocodile (Crocodylus moreletii) from a transboundary river between Mexico and Belize: Archives of Environmental Contamination and Toxicology, 68(2), 265-273.

Chandrasekaran, A., Ravisankar, R., 2015, Spatial distribution of physicochemical properties and function of heavy metals in soils of Yelagiri hills, Tamilnadu by energy dispersive X-ray fluorescence spectroscopy (EDXRF) with statistical approach: Spectrochimica Acta, Part A: Molecular and Biomolecular Spectroscopy, 150, 586-601.

Cheraghi, M., Lorestani, B., Merrikhpour, H., 2012, Investigation of the effects of Phosphate Fertilizer Application on the Heavy Metal Content in Agricultural Soils with Different Cultivation Patterns: Biological Trace Element Research, 145, 87-92.

Clemente, R., Dickinson, M.N., Lepp, W.N., 2008, Mobility of metals and metalloids in a multi-element contaminated soil 20 years after cessation of the pollution source activity: Environmental Pollution, 155, 254-261.

CICOPLAFEST (Comisión Intersecretarial para el Control del Proceso y Uso de Plaguicidas, Fertilizantes y Sustancias Tóxicas), SESA (Secretaría de Salud), SAGARPA (Secretaría de Agricultura, Ganadería, Desarrollo Rural, Pesca y Alimentación), SEMARNAT (Secretaría del Medio Ambiente y Recurso Naturales), 2004, Catálogo de plaguicidas, (en línea), disponible en: http:// www.cofepris.gob.mx/AZ/Paginas/Plaguicidas\%20y\%20Fertilizantes/ CatalogoPlaguicidas.aspx, consulta: 1 de abril de 2016.

CONAGUA (Comisión Nacional del Agua), 2010, Regiones Hidrológicas, (en línea), disponible en: <http://www.conagua.gob.mx/atlas/ciclo09.html>, consulta: 9 de septiembre de 2016.

De la Cruz-Pons, A., Zavala-Cruz, J., Guerrero-Peña, A., Salgado-García, S., Lagunes-Espinoza, L.C., Gavi-Reyes, F., 2012, Metales pesados en suelos cultivados con caña de azúcar en la Chontalpa, Tabasco: Universidad y Ciencia, 28(2), 119-130.

De Matos, A.T., Montes, M.P., Da Costa, L.M., Martínez, M.A., 2001, Mobility of heavy metals as related to soil chemical and mineralogical characteristics of Brazilian soils: Environmental Pollution, 111, 429-435.

Doménech, X., Peral, J., 2006, Comportamiento y destino de los contaminantes en los sistemas terrestres, en Química Ambiental de Sistemas Terrestres: Barcelona, Reverté S.A. de C.V., 119-180.

Dube, A., Zbyniewski, R., Kowalkowski, T., Buszewski, B., 2001, Adsorption and Migration of heavy metals in soil: Polish Journal of Environmental Studies, 10(1), 1-10.

El Vergel de Occidente S.A de C.V., s.f., Ficha técnica dinactiv, (en línea), disponible en: http://www.agrovergel.com/fichas/dinactiv.pdf; consulta: 1 de abril de 2016.

FAO (Food and Agricultural Organization), 2014, World reference base for soil resources, International soil classification system for naming soils and creating legends for soil maps 2014: Roma, Italia, World Soil Resources Reports, 106, $181 \mathrm{pp}$.

FAO (Food and Agricultural Organization), 2017, Conservación de los recursos naturales para una agricultura sostenible, (en línea), disponible en: http:// www.fao.org/ag/ca/training_materials/cd27-spanish/tme/tools.pdf.>; consulta: 19 de mayo de 2017.

FAR (Foundation of Agronomic Research), PPIC (Potash and Phosphate Institute of Canada), 1988, Conceptos de fertilidad y productividad de suelos, en Manual de fertilidad de los suelos: Canadá, Potash and Phosphate Institute, Manual técnico, 6-15.

Fernández-Pérez, L., Leal-Bautista, R.M., 2013, Informe de la segunda etapa del proyecto "Determinación de agroquímicos lixiviados en la zona cañera de Quintana Roo": Chetumal, México, Comisión de cuenca de Río Hondo, (Informe), $62 \mathrm{pp}$.

Gimeno-García, E., Andreu, V., Boluda, R., 1995, Heavy metals incidence in 
the application of inorganic fertilizers and pesticides to rice farming soils: Environmental Pollution, 92(1), 19-25.

Herrera, J., 2011, Hidrología Subterránea, en Pozo, C., Armilio-Canto, N., Calme, S. (eds.), Riqueza Biológica de Quintana Roo, un análisis para su conservación: México, D.F., Comisión Nacional para el Conocimiento y Uso de la Biodiversidad, El Gobierno del estado de Quintana Roo, El Colegio de la Frontera Sur, Programa de Pequeñas Donaciones--México, 34-41.

INEGI (Instituto Nacional de Estadística, Geografía e Informática), 2013a, Simulador de Flujos de Agua de Cuencas Hidrográficas (SIATL) 3.1, (en línea), disponible en: http://antares.inegi.org.mx/analisis/red_hidro/ SIATL/\#; consulta: 13 de abril de 2016.

INEGI (Instituto Nacional de Estadística, Geografía e Informática), 2013b, Continuo de Elevaciones Mexicano 3.0 (CEM 3.0), (en línea), disponible en: http://www.inegi.org.mx/geo/contenidos/datosrelieve/continental/ continuoelevaciones.aspx; consulta: 25 de febrero de 2016.

Iuit-Jiménez, A.C., 2017, Desafíos socio-ambientales y uso de agroquímicos en la región cañera de Río Hondo en el sur de México: Chetumal, Quintana Roo, El Colegio de la Frontera Sur, tesis de maestría, 65 pp.

Kennou, B., El Meray, M., Romane, A., Arjouni, Y., 2015, Assessment of heavy metal availability $(\mathrm{Pb}, \mathrm{Cu}, \mathrm{Cr}, \mathrm{Cd}, \mathrm{Zn})$ and speciation in contaminated soils and sediment of discharge by sequential extraction: Environmental Earth Science, 74(7), 5849-5858.

Mangum, S.J., 2009, Microwave Digestion EPA Method 3052 on the Multiwave 3000 (en línea), disponible en: http://www.perkinelmer.com.cn/Content/ ApplicationNotes/APP_MicrowaveDigestionMultiwave.pdf; consulta: 13 de abril de 2016.

McLean, J.E., Bledsoe, B.E., 1992, Behavior of metals in soils, en Ground Water Issue (en línea), disponible en: https:/www.epa.gov/sites/production/ files/2015-06/documents/issue_behavior_metals_soil.pdf; consulta: 17 de abril de 2016.

Moore, D.S., 2005, Estadística Aplicada Básica, Bosche, A. (ed.), España, Antoni Bosche S.A., 874 pp.

Murdock, D., 2014, R for Windows, (en línea), ver. 3.1.3, https://cran.r-project. org/bin/windows/base/old/3.1.3/; consulta: noviembre de 2016 .

Palau-Bioquim S.A. de C.V., s.f., Ficha técnica Algaenzims, (en línea), disponible en: http://www.agrolahuerta.com.mx/intranet/mod/PLM/DIPO/src/ productos/103_28.htm consulta: 8 de abril de 2016.

Palma-López, D.J., Salgado-García, S., Obrador-Olán, J.J., Trujillo-Narcía, A., Lagunes-Espinoza, L. del C., Zavala-Cruz, J., Ruíz-Bello, A., CarreraMartel, M.A., 2002, Sistema Integrado para recomendar dosis de fertilización en caña de azúcar (SIRDF): Terra Latinoamericana, 20(3), 347-358.

Prieto-Méndez, J., González-Ramírez, C.A., Román-Gutiérrez, A.D., PrietoGarcía, F., 2009, Contaminación y fitotoxicidad en plantas por metales pesados provenientes de suelos y agua: Tropical and Subtropical Agroecosystems, 10(1), 29-44.

Rainwater, T.R., Wu, T.H., Finger, A.G., Cañas, J.E., Yu, L., Reynolds, K.D., Coimbatore, G., Barr, B., Platt, S.G., Cobb, G.P., Anderson, T.A., McMurry, S.T., 2007, Metals and organochlorine pesticides in caudal scutes of crocodiles from Belize and Costa Rica: The Science of the Total Environment, 373(1), 146-156.

Romero-Calderón, A.G., Morales-Vela, B., Rosíles-Martínez, R., OliveraGómez, L.D., Delgado-Estrella, A., 2016, Metals in bone tissue of antillan manatees from the gulf of México and Chetumal bay, México: Bulletin of Environmental Contamination and Toxicology, 96(1), 9-14

Salgado-García, S., Núñez-Escobar, R., Peña-Cabriales, J.J., Etchvers-Barra, J.D., Palma-López, D.J., Soto-Hernández, M.R., 2000, Respuesta de la soca de caña de azúcar a la fertilización NPK: Agrociencia, 34(6), 689-698.
Sánchez-Pool, N.E., 2007, Análisis espacio-temporal de las concentraciones de metales pesados ( $\mathrm{Cd}, \mathrm{Cu}, \mathrm{Fe}, \mathrm{Pb}$ y $\mathrm{Zn}$ ) en columna de agua, sedimento y en las macroalgas Batophora spp. y Bostrychia spp. en la Bahía de Chetumal, Quintana Roo: Chetumal, México, Universidad de Quintana Roo, tesis de licenciatura, $96 \mathrm{pp}$.

Sánchez-Sánchez, J.A., Álvarez-Legorreta, T., Pacheco-Ávila, J.G., González-Herrera, R.A., Carrillo-Bribiezca, L., 2015, Caracterización hidrogeoquímica de las aguas subterráneas del sur del Estado de Quintana Roo, México: Revista Mexicana de Ciencias Geológicas, 32(1), 62-76.

SEMARNAT (Secretaría de Medio Ambiente y Recursos Naturales), 2002, Norma oficial mexicana NOM-021-SEMARNAT-2000 que establece las especificaciones de fertilidad, salinidad y clasificación de suelos, estudio, muestreo y análisis, México: Diario Oficial de la Federación, 82 pp.

Segura-Muñoz, S.I., da-Silva, O.A., Nikaido, M., Trevilato, T.M., Bocio, A., Takayanaqui, A.M., Domingo, J.L., 2005, Metal levels in sugar cane (Saccharum spp.) samples from an area under the influence of a municipal landfill and a medical waste treatment system in Brazil: Environmental International, 32(1), 52-57.

Sherene, T., 2010, Mobility and transport of heavy metals in polluted soil environment: Biological Forum-An International Journal, 2(2), 112-121.

SIAP (Servicio de Información y Estadística Agroalimentaria y Pesquera), 2014 Cierre de la producción agrícola por cultivo (en línea), disponible en: http://www.siap.gob.mx/cierre-de-la-produccion-agricola-por-cultivo/; consulta: 23 de agosto de 2015 .

Storer, A.D., 1984. A simple high volume ashing procedure for determining soil organic matter: Communications in Soil Science and Plant Analysis, 15(7), 759-772.

Tessier, A., Campbell, P., Bisson, M., 1979, Sequential Extraction Procedure for the Speciation of Particulate Trace Metals: Analytical Chemistry, 51(7), 844-851.

Ungureanu, T., Ovidiu Iancu, G., Pintilei, M., Marius Chicos, M., 2016, Spatial distribution and geochemistry of heavy metals in soils: A case study from the NE area of Vaslui country, Romania: Journal of Chemical Exploration, 13 pp., http://dx.doi.org/10.1016/j.gexplo.2016.08.012.

VELSIMEX, S.A. de C.V., 2009, Ficha técnica Velconate 480 (En línea), disponible en: http://www.velsimex.com/pdfs/Velconate480x.pdf; consulta: 31 marzo 2016.

Violante, A., Cozzolino, V., Perelomov, L., Caporale, A.G., Pigna, M., 2010, Mobility and bioavailability of heavy metals and metalloids in soil environments: Journal of Soil Science Plant Nutrition, 10(3), 268-292.

Wang, Q., Xie, Z., Li, F., 2015, Using ensemble models to identify and apportion heavy metals pollution sources in agricultural soils on a local scale: Environmental Pollution, 206, 227-235.

Wen-Huang, S., Yun-Jin, J., 2008, Status of heavy metals in agricultural soils as affected by different patterns of land use: Environmental Monitoring and Assessment, 139, 317-327.

Yadav, D., Jain, R., Rai, R., 2010, Impact of heavy metals on sugarcane, en Sherameti, I., Varma, A. (eds.), Soil Biology: Berlin, Springer-Verlag, 339-368.

Manuscrito recibido: abril 11, 2016

Manuscrito coregido recibido: mayo 19, 2017

Manuscrito aceptado: junio 3, 2017 